\title{
HESSIAN ESTIMATES FOR SPECIAL LAGRANGIAN EQUATIONS WITH CRITICAL AND SUPERCRITICAL PHASES IN GENERAL DIMENSIONS
}

\author{
DAKE WANG AND YU YUAN
}

\begin{abstract}
We derive a priori interior Hessian estimates for special Lagrangian equation with critical and supercritical phases in general higher dimensions. Our unified approach leads to sharper estimates even for the previously known three dimensional and convex solution cases.
\end{abstract}

\section{INTRODUCTION}

In this paper, we complete a priori interior Hessian estimates for the special Lagrangian equation

$$
\sum_{i=1}^{n} \arctan \lambda_{i}=\Theta
$$

with critical and supercritical phases $|\Theta| \geq(n-2) \pi / 2$ in all dimensions $n \geq 3$, where $\lambda=\left(\lambda_{1}, \cdots, \lambda_{n}\right)$ are the eigenvalues of the Hessian $D^{2} u$. For solutions to (1.1) with $|\Theta| \geq(n-2) \pi / 2$ in dimension two and three, and also convex solutions to (1.1) in all dimensions, Hessian estimates have been obtained in [WY2,3,4] and [CWY].

Equation (1.1) originates in the special Lagrangian geometry by HarveyLawson [HL]. The Lagrangian graph $(x, D u(x)) \subset \mathbb{R}^{n} \times \mathbb{R}^{n}$ is called special when the argument of the complex number $\left(1+\sqrt{-1} \lambda_{1}\right) \cdots\left(1+\sqrt{-1} \lambda_{n}\right)$ or the phase is constant $\Theta$, and it is special if and only if $(x, D u(x))$ is a (volume minimizing) minimal surface in $\mathbb{R}^{n} \times \mathbb{R}^{n}$ [HL, Theorem 2.3, Proposition 2.17]. The phase $(n-2) \pi / 2$ is called critical because the level set $\left\{\lambda \in \mathbb{R}^{n} \mid \lambda\right.$ satisfying (1.1) $\}$ is convex only when $|\Theta| \geq(n-2) \pi / 2[\mathrm{Y} 2$, Lemma 2.1]. The algebraic form of (1.1) is

$$
\cos \Theta \sum_{1 \leq 2 k+1 \leq n}(-1)^{k} \sigma_{2 k+1}-\sin \Theta \sum_{0 \leq 2 k \leq n}(-1)^{k} \sigma_{2 k}=0,
$$

where $\sigma_{k}$ s are the elementary symmetric functions of the Hessian $D^{2} u$.

We state our main result in the following.

Date: October 20, 2018.

Both authors are partially supported by an NSF grant. 
Theorem 1.1. Let $u$ be a smooth solution to (1.1) with $|\Theta| \geq(n-2) \pi / 2$ and $n \geq 3$ on $B_{R}(0) \subset \mathbb{R}^{n}$. Then we have

$$
\left|D^{2} u(0)\right| \leq C(n) \exp \left[C(n) \max _{B_{R}(0)}|D u|^{2 n-2} / R^{2 n-2}\right] ;
$$

and when $|\Theta|=(n-2) \pi / 2$, we also have

$$
\left|D^{2} u(0)\right| \leq C(n) \exp \left[C(n) \max _{B_{R}(0)}|D u|^{2 n-4} / R^{2 n-4}\right] .
$$

Relying on our previous gradient estimates for (1.1) with $|\Theta| \geq(n-2) \pi / 2$ in [WY4]

$$
\max _{B_{R}(0)}|D u| \leq C(n)\left[\underset{B_{2 R}(0)}{\operatorname{osc}} \frac{u}{R}+1\right],
$$

we can bound $D^{2} u$ in terms of the solution $u$ in $B_{2 R}(0)$.

Singular solutions to (1.1) with subcritical phases $|\Theta|<(n-2) \pi / 2$ and $n \geq 3$ constructed by Nadirashvili-Vlădut [NV] and the authors [WdY] show that the critical and supercritical phase condition in Theorem 1.1 is necessary.

One application of the above estimates is the regularity (analyticity) of the $C^{0}$ viscosity solutions to (1.1) with $|\Theta| \geq(n-2) \pi / 2$. Another quick consequence is a Liouville type result for global solutions with quadratic growth to (1.1) with $|\Theta|=(n-2) \pi / 2$, namely any such a solution must be quadratic (cf. [Y1], [Y2] where other Liouville type results for convex solutions to (1.1) and Bernstein type results for global solutions to (1.1) with supercritical phase $|\Theta|>(n-2) \pi / 2$ were obtained $)$.

In the 1950's, Heinz $[\mathrm{H}]$ derived a Hessian bound for the two dimensional Monge-Ampère type equation including (1.1) with $n=2$; see also Pogorelov [P1] for Hessian estimates for these equations including (1.1) with $|\Theta|>\pi / 2$ and $n=2$. In the 1970's Pogorelov [P2] constructed his famous counterexamples, namely irregular solutions to three dimensional MongeAmpère equations $\sigma_{3}\left(D^{2} u\right)=\operatorname{det}\left(D^{2} u\right)=1$; those irregular solutions also serve as counterexamples for cubic and higher order symmetric $\sigma_{k}$ equations (cf. [U1]). In passing, we also mention Hessian estimates for solutions with certain strict convexity constraints to Monge-Ampère equations and $\sigma_{k}$ equation $(k \geq 2)$ by Pogorelov [P2] and Chou-Wang [CW] respectively using the Pogorelov technique. Trudinger [T2] and Urbas [U2][U3], also Bao-Chen $[\mathrm{BC}]$ obtained (pointwise) Hessian estimates in terms of certain integrals of the Hessian, for $\sigma_{k}$ equations and special Lagrangian equation (1.1) with $n=3, \Theta=\pi$ respectively. Pointwise Hessian estimates for strictly convex solutions to quotient equations $\sigma_{n} / \sigma_{k}$ were derived in terms of certain integrals of the Hessian by Bao-Chen-Guan-Ji [BCGJ].

Our strategies for the Hessian estimates go as follows. We bound the subharmonic function of the Hessian $b=\ln \sqrt{1+\lambda_{\max }^{2}}$ by its integral on the minimal surface using Michael-Simon's mean value inequality [MS]. Applying certain Sobolev inequalities, we estimate the integral of $b$ by the integral 
of its gradient. The decisive choice $b$ satisfies a Jacobi inequality: its Laplacian bounds its gradient; in turn, the integral of the gradient $b$ is bounded by a weighted volume of the minimal Lagrangian graph. By a conformality identity, the weighted volume element is in fact the trace of the linearized operator of the special Lagrangian equation in algebraic form, which is a linear combination of the elementary symmetric functions of the Hessian. Taking advantage of the divergence structure of those functions, we bound the weighted volume in terms of the height of special Lagrangian graph, or the gradient of the solution.

However, there are two major difficulties in the execution for general dimension. The first one is to justify the nonlinear Jacobi inequality in the integral sense for the Lipschitz only function $b$, which was only achieved in dimension three by involved arguments [WY2]. The second one is to find, in the critical phase case, a relative isoperimetric inequality or equivalent Sobolev inequality for functions without compact support, which was circumvented only in dimension three thanks to the linear dependence on the Hessian for the linearized operator of now equivalent equation $\sigma_{2}=1$ [WY2]. We overcome the first one by observing that the Jacobi inequality and its equivalent linear formulation hold in the viscosity sense, consequently in the potential sense. By Hervé-Hervé [HH, Theorem 1] (see also Watson [Wn, p. 246]), the linear inequality holds in the integral sense, in turn, so does the needed Jacobi inequality. Conceptually it is natural this way. For details, see the proof of Proposition 2.1. To deal with the second difficulty, we instead apply the Sobolev inequality for functions with compact supports, but use a "twist-multiplication" trick to contain the terms involving derivatives of the cut-off functions (Step 4 in Section 3). This trick enables us to have a unified approach (for both the critical and supercritical cases) in all dimensions $n \geq 3$. Even in the known three dimensional [WY2,4] and convex cases [CWY], the simpler unified argument leads to sharper Hessian estimates.

Our unified arguments does not work for (1.1) with $\Theta=0$ and $n=2$, as the Jacobi inequality fails (only) for harmonic functions. Elementary methods in [WY3] led to the sharp Hessian estimates in dimension two. (The sharp Hessian estimates in terms of the linear exponential dependence on the gradients, can be seen by the corresponding solutions to the MongeAmpère equation or (1.1) with $\Theta=\pi / 2$ and $n=2$, converted from Finn's minimal surface $[\mathrm{F}$, p. 355] via Heinz transformation [J, p. 133].)

As one can see that, not only our Hessian-slope estimates for "gradient" minimal graphs are analogous to the gradient-slope estimates for the codimension one minimal graphs, but also our arguments resemble the original integral proof by Bombieri-De Giorgi-Miranda [BDM] and the simplified one by Trudinger [T1] for the latter classical result. When one tries to adapt the later Korevaar pointwise technique $[\mathrm{K}]$, certain extra structure or assumption has to be used, as in [WY1]. Otherwise, an adaptation of the technique alone would lead to Hessian estimates for the Monge-Ampère equations, 
to which the Jacobi inequality is available. But this is inconsistent with Pogorelov's singular solutions [P2].

Notation. First $\partial_{i}=\frac{\partial}{\partial_{x_{i}}}, \partial_{i j}=\frac{\partial^{2}}{\partial x_{i} \partial x_{j}}, u_{i}=\partial_{i} u=D_{i} u, u_{j i}=\partial_{i j} u$ etc., but $\lambda_{1}, \cdots, \lambda_{n}$ and $b_{k}=\left(\ln \sqrt{1+\lambda_{1}^{2}}+\cdots+\ln \sqrt{1+\lambda_{k}^{2}}\right) / k$ do not represent the partial derivatives. Also

$$
\sigma_{k}\left(\lambda_{1}, \cdots, \lambda_{n}\right)=\sum_{1 \leq i_{1}<\cdots<i_{k} \leq n} \lambda_{i_{1}} \cdots \lambda_{i_{k}}
$$

Further, $h_{i j k}$ will denote (the second fundamental form)

$$
h_{i j k}=\frac{1}{\sqrt{1+\lambda_{i}^{2}}} \frac{1}{\sqrt{1+\lambda_{j}^{2}}} \frac{1}{\sqrt{1+\lambda_{k}^{2}}} u_{i j k} .
$$

when $D^{2} u$ is diagonalized. Finally $C(n)$ will denote various constants depending only on dimension $n$.

\section{Preliminary inequalities}

Taking the gradient of both sides of the special Lagrangian equation (1.1), we have

$$
\sum_{i, j=1}^{n} g^{i j} \partial_{i j}(x, D u(x))=0
$$

where $\left(g^{i j}\right)$ is the inverse of the induced metric $g=\left(g_{i j}\right)=I+D^{2} u D^{2} u$ on the surface $(x, D u(x)) \subset \mathbb{R}^{n} \times \mathbb{R}^{n}$. Simple geometric manipulation of (2.1) yields the usual form of the minimal surface equation

$$
\triangle_{g}(x, D u(x))=0
$$

where the Laplace-Beltrami operator of the metric $g$ is given by

$$
\triangle_{g}=\frac{1}{\sqrt{\operatorname{det} g}} \sum_{i, j=1}^{n} \partial_{i}\left(\sqrt{\operatorname{det} g} g^{i j} \partial_{j}\right) .
$$

Because we are using harmonic coordinates $\triangle_{g} x=0$, we see that $\triangle_{g}$ also equals the linearized operator of the special Lagrangian equation (1.1) at $u$,

$$
\triangle_{g}=\sum_{i, j=1}^{n} g^{i j} \partial_{i j}
$$


The volume form, gradient and inner product with respect to the metric $g$ are

$$
\begin{aligned}
d v_{g} & =\sqrt{\operatorname{det} g} d x \\
\nabla_{g} v & =\left(\sum_{k=1}^{n} g^{1 k} v_{k}, \cdots, \sum_{k=1}^{n} g^{n k} v_{k}\right), \\
\left\langle\nabla_{g} v, \nabla_{g} w\right\rangle_{g} & =\sum_{i, j=1}^{n} g^{i j} v_{i} w_{j}, \text { in particular }\left|\nabla_{g} v\right|^{2}=\left\langle\nabla_{g} v, \nabla_{g} v\right\rangle_{g} .
\end{aligned}
$$

We begin with some algebraic and trigonometric inequalities needed in this paper.

Lemma 2.1. Suppose the ordered real numbers $\lambda_{1} \geq \lambda_{2} \geq \cdots \geq \lambda_{n}$ satisfy (1.1) with $\Theta \geq(n-2) \pi / 2$ and $n \geq 2$. Then we have

$$
\begin{gathered}
\lambda_{1} \geq \lambda_{2} \geq \cdots \geq \lambda_{n-1}>0 \text { and } \lambda_{n-1} \geq\left|\lambda_{n}\right|, \\
\lambda_{1}+(n-1) \lambda_{n} \geq 0, \\
\sigma_{k}\left(\lambda_{1}, \cdots, \lambda_{n}\right) \geq 0 \text { for all } 1 \leq k \leq n-1 .
\end{gathered}
$$

Proof. Set $\theta_{i}=\arctan \lambda_{i}$. Property (2.2) follows from the inequalities

$$
\theta_{n-1}+\theta_{n} \geq(n-2) \pi / 2-\left(\theta_{1}+\cdots+\theta_{n-2}\right) \geq 0 .
$$

We only need to check property (2.3) when $\lambda_{n}<0$ or $\theta_{n}<0$. We know

$$
\frac{\pi}{2}>\frac{\pi}{2}+\theta_{n} \geq\left(\frac{\pi}{2}-\theta_{1}\right)+\cdots+\left(\frac{\pi}{2}-\theta_{n-1}\right)>0 .
$$

It follows that

$$
\begin{aligned}
-\frac{1}{\lambda_{n}} & =\tan \left(\frac{\pi}{2}+\theta_{n}\right) \\
& \geq \tan \left(\frac{\pi}{2}-\theta_{1}\right)+\cdots+\tan \left(\frac{\pi}{2}-\theta_{n-1}\right)=\frac{1}{\lambda_{1}}+\cdots+\frac{1}{\lambda_{n-1}} \\
& \geq(n-1) \frac{1}{\lambda_{1}} .
\end{aligned}
$$

Then we get (2.3).

Next we prove property (2.4) with $k=n-1$. We only need to deal with the case $\lambda_{n}<0$. From (2.5), we have

$$
0 \geq \frac{1}{\lambda_{1}}+\cdots+\frac{1}{\lambda_{n-1}}+\frac{1}{\lambda_{n}}=\frac{\sigma_{n-1}\left(\lambda_{1}, \cdots, \lambda_{n}\right)}{\left(\lambda_{1} \cdots \lambda_{n-1}\right) \lambda_{n}} .
$$

Using $\lambda_{1} \geq \lambda_{2} \geq \cdots \geq \lambda_{n-1}>0>\lambda_{n}$, we get $\sigma_{n-1}\left(\lambda_{1}, \cdots, \lambda_{n}\right) \geq 0$.

Finally we prove the whole property (2.4) inductively. Property (2.4) with $n=2$ is obvious (or by the above). Assume property (2.4) with $n=m$ is true, that is

$$
\sigma_{j}\left(\lambda_{1}, \cdots, \lambda_{m}\right) \geq 0 \text { for } 1 \leq j \leq m-1,
$$

provided $\arctan \lambda_{1}+\cdots+\arctan \lambda_{m} \geq(m-2) \pi / 2$. 
Let us prove (2.4) with $n=m+1$ for

$$
\arctan \lambda_{1}+\cdots+\arctan \lambda_{m+1} \geq(m-1) \pi / 2 \text {. }
$$

By the proved property (2.4) with $k=n-1=m$, we get $\sigma_{m}\left(\lambda_{1}, \cdots, \lambda_{m+1}\right) \geq$ 0 . We only need to verify the other $\sigma$ inequalities when the smallest number is negative, say $\lambda_{1} \geq \lambda_{2} \geq \cdots \geq \lambda_{m}>0>\lambda_{m+1}$. (By (2.2), only the smallest $\lambda_{m+1}$ can be negative.) We have

$$
\sigma_{m-1}\left(\lambda_{1}, \cdots, \lambda_{m+1}\right)=\sigma_{m-1}\left(\lambda_{2}, \cdots, \lambda_{m+1}\right)+\lambda_{1} \sigma_{m-2}\left(\lambda_{2}, \cdots, \lambda_{m+1}\right) .
$$

From (2.6), we infer

$$
\arctan \lambda_{2}+\cdots+\arctan \lambda_{m+1} \geq(m-2) \pi / 2 .
$$

By the induction assumption, we should have

$$
\sigma_{m-1}\left(\lambda_{2}, \cdots, \lambda_{m+1}\right) \geq 0 \text { and } \sigma_{m-2}\left(\lambda_{2}, \cdots, \lambda_{m+1}\right) \geq 0 .
$$

Thus we obtain $\sigma_{m-1}\left(\lambda_{1}, \cdots, \lambda_{m+1}\right) \geq 0$. Similarly we prove $\sigma_{i}\left(\lambda_{1}, \cdots, \lambda_{m+1}\right) \geq$ 0 for $1 \leq i \leq m-2$. Therefore property (2.4) holds for all $n \geq 2$. This completes the proof of Lemma 2.1.

Lemma 2.2. Let $u$ be a smooth solution to (1.1). Suppose that the Hessian $D^{2} u$ is diagonalized and the eigenvalue $\lambda_{\gamma}$ is distinct from all other eigenvalues of $D^{2} u$ at point $p$. Then we have at $p$

$$
\left|\nabla_{g} \ln \sqrt{1+\lambda_{\gamma}^{2}}\right|^{2}=\sum_{k=1}^{n} \lambda_{\gamma}^{2} h_{\gamma \gamma k}^{2}
$$

and

$$
\begin{aligned}
& \triangle_{g} \ln \sqrt{1+\lambda_{\gamma}^{2}}= \\
& \left(1+\lambda_{\gamma}^{2}\right) h_{\gamma \gamma \gamma}^{2}+\sum_{k \neq \gamma}\left(\frac{2 \lambda_{\gamma}}{\lambda_{\gamma}-\lambda_{k}}+\frac{2 \lambda_{\gamma}^{2} \lambda_{k}}{\lambda_{\gamma}-\lambda_{k}}\right) h_{k k \gamma}^{2} \\
& +\sum_{k \neq \gamma}\left[1+\frac{2 \lambda_{\gamma}}{\lambda_{\gamma}-\lambda_{k}}+\frac{\lambda_{\gamma}^{2}\left(\lambda_{\gamma}+\lambda_{k}\right)}{\lambda_{\gamma}-\lambda_{k}}\right] h_{\gamma \gamma k}^{2} \\
& +\sum_{\substack{k>j \\
k, j \neq \gamma}} 2 \lambda_{\gamma}\left[\frac{1+\lambda_{k}^{2}}{\lambda_{\gamma}-\lambda_{k}}+\frac{1+\lambda_{j}^{2}}{\lambda_{\gamma}-\lambda_{j}}+\left(\lambda_{j}+\lambda_{k}\right)\right] h_{k j \gamma}^{2} .
\end{aligned}
$$

Proof. The calculation was done in Lemma 2.1 of [WY2].

Lemma 2.3. Let $u$ be a smooth solution to (1.1) with $\Theta \geq(n-2) \frac{\pi}{2}$. Suppose that the ordered eigenvalues $\lambda_{1} \geq \lambda_{2} \geq \cdots \geq \lambda_{n}$ of the Hessian $D^{2} u$ satisfy $\lambda_{1}=\cdots=\lambda_{m}>\lambda_{m+1}$ at point $p$. Then the function $b_{m}=\frac{1}{m} \sum_{i=1}^{m} \ln \sqrt{1+\lambda_{i}^{2}}$ is smooth near $p$ and satisfies at $p$

$$
\triangle_{g} b_{m} \geq\left(1-\frac{4}{\sqrt{4 n+1}+1}\right)\left|\nabla_{g} b_{m}\right|^{2} .
$$


Proof. Step 1. The function $b_{m}$ is symmetric in $\lambda_{1}, \cdots, \lambda_{m}$. Thus for $m<n$, $b_{m}$ is smooth when $\lambda_{m}>\lambda_{m+1}$, in particular near $p$, at which $\lambda_{1}=\cdots=$ $\lambda_{m}>\lambda_{m+1}$. For $m=n, b_{n}$ is certainly smooth everywhere.

We again assume that Hessian $D^{2} u$ is diagonalized at point $p$. Let us also first assume the first $m$ eigenvalues $\lambda_{1}, \cdots, \lambda_{m}$ are distinct. Using (2.8) in Lemma 2.2, we calculate $\triangle_{g} b_{m}$; after grouping those terms $h_{\diamond \diamond \diamond}, h_{\odot \odot}$ and $h_{\odot} \diamond$ in the summation, we obtain

$$
\begin{aligned}
& m \triangle_{g} b_{m}=\sum_{\gamma=1}^{m} \triangle_{g} \ln \sqrt{1+\lambda_{\gamma}^{2}} \stackrel{p}{=} \\
& \sum_{k \leq m}\left(1+\lambda_{k}^{2}\right) h_{k k k}^{2}+\left(\sum_{i<k \leq m}+\sum_{k<i \leq m}\right)\left(3+\lambda_{i}^{2}+2 \lambda_{i} \lambda_{k}\right) h_{i i k}^{2}+\sum_{k \leq m<i} \frac{2 \lambda_{k}\left(1+\lambda_{k} \lambda_{i}\right)}{\lambda_{k}-\lambda_{i}} h_{i i k}^{2} \\
& +\sum_{i \leq m<k} \frac{3 \lambda_{i}-\lambda_{k}+\lambda_{i}^{2}\left(\lambda_{i}+\lambda_{k}\right)}{\lambda_{i}-\lambda_{k}} h_{i i k}^{2}+ \\
& \left\{\begin{array}{l}
2 \sum_{i<j<k \leq m}\left(3+\lambda_{i} \lambda_{j}+\lambda_{j} \lambda_{k}+\lambda_{k} \lambda_{i}\right) h_{i j k}^{2}+ \\
2 \sum_{i<j \leq m<k}\left(1+\lambda_{i} \lambda_{j}+\lambda_{j} \lambda_{k}+\lambda_{k} \lambda_{i}+\lambda_{i} \frac{1+\lambda_{k}^{2}}{\lambda_{i}-\lambda_{k}}+\lambda_{j} \frac{1+\lambda_{k}^{2}}{\lambda_{j}-\lambda_{k}}\right) h_{i j k}^{2}+. \\
2 \sum_{i \leq m<j<k} \lambda_{i}\left[\lambda_{j}+\lambda_{k}+\frac{1+\lambda_{j}^{2}}{\lambda_{i}-\lambda_{j}}+\frac{1+\lambda_{k}^{2}}{\lambda_{j}-\lambda_{k}}\right] h_{i j k}^{2}
\end{array}\right.
\end{aligned}
$$

Now as a function of the matrices (then composed with smooth matrix function $D^{2} u$ of $\left.x\right), b_{m}$ is $C^{2}$ at $D^{2} u(p)$ with eigenvalues satisfying $\lambda=$ $\lambda_{1}=\cdots=\lambda_{m}>\lambda_{m+1}$. Note that $D^{2} u(p)$ can be approximated by matrices with distinct eigenvalues. Therefore the above expression for $\triangle_{g} b_{m}$ at $p$ still holds and simplifies to

$$
\begin{aligned}
& m \triangle_{g} b_{m} \underline{\underline{p}} \\
& \sum_{k \leq m}\left(1+\lambda^{2}\right) h_{k k k}^{2}+\left(\sum_{i<k \leq m}+\sum_{k<i \leq m}\right)\left(3+3 \lambda^{2}\right) h_{i i k}^{2}+\sum_{k \leq m<i} \frac{2 \lambda\left(1+\lambda \lambda_{i}\right)}{\lambda-\lambda_{i}} h_{i i k}^{2}+ \\
& \sum_{i \leq m<k} \frac{3 \lambda-\lambda_{k}+\lambda^{2}\left(\lambda+\lambda_{k}\right)}{\lambda-\lambda_{k}} h_{i i k}^{2}+ \\
& \left\{\begin{array}{l}
2 \sum_{i<j<k \leq m}\left(3+3 \lambda^{2}\right) h_{i j k}^{2}+ \\
2 \sum_{i<j \leq m<k}\left[1+\frac{2 \lambda}{\lambda-\lambda_{k}}+\frac{\lambda^{2}\left(\lambda+\lambda_{k}\right)}{\lambda-\lambda_{k}}\right] h_{i j k}^{2}+ \\
2 \sum_{i \leq m<j<k} \lambda\left(\lambda_{j}+\lambda_{k}+\frac{1+\lambda_{j}^{2}}{\lambda-\lambda_{j}}+\frac{1+\lambda_{k}^{2}}{\lambda-\lambda_{k}}\right) h_{i j k}^{2} \\
\geq \sum_{k \leq m} \lambda^{2} h_{k k k}^{2}+\left(\sum_{i<k \leq m}+\sum_{k<i \leq m}\right) 3 \lambda^{2} h_{i i k}^{2}+\sum_{k \leq m<i} \frac{2 \lambda^{2} \lambda_{i}}{\lambda-\lambda_{i}} h_{i i k}^{2}+ \\
\sum_{i \leq m<k} \frac{\lambda^{2}\left(\lambda+\lambda_{k}\right)}{\lambda-\lambda_{k}} h_{i i k}^{2},
\end{array}\right.
\end{aligned}
$$

where we used (2.2) of Lemma 2.1 in the inequality. 
Similarly by (2.7) in Lemma 2.2 and the $C^{1}$ continuity of $b_{m}$ as a function of matrices at $D^{2} u(p)$, we obtain

$$
\left|\nabla_{g} b_{m}\right|^{2} \stackrel{p}{=} \frac{1}{m^{2}} \sum_{1 \leq k \leq n} \lambda^{2}\left(\sum_{i \leq m} h_{i i k}\right)^{2} \leq \frac{\lambda^{2}}{m} \sum_{1 \leq k \leq n}\left(\sum_{i \leq m} h_{i i k}^{2}\right)
$$

From the above two inequalities, it follows that

$$
\begin{gathered}
m\left(\triangle_{g} b_{m}-\varepsilon\left|\nabla_{g} b_{m}\right|^{2}\right) \geq \\
\lambda^{2}\left[\begin{array}{c}
\sum_{k \leq m}(1-\varepsilon) h_{k k k}^{2}+\left(\sum_{i<k \leq m}+\sum_{2 \lambda}+\sum_{k<i \leq m}\right)(3-\varepsilon) h_{i i k}^{2} \\
+\sum_{k \leq m<i} \frac{\lambda-\lambda_{i}}{2} h_{i i k}
\end{array}\right]+ \\
\lambda^{2}\left[\sum_{i \leq m<k}\left(\frac{\lambda+\lambda_{k}}{\lambda-\lambda_{k}}-\varepsilon\right) h_{i i k}^{2}\right]
\end{gathered}
$$

with $\varepsilon$ to be fixed.

Step 2. We show (2.10) and (2.11) in the above inequality are nonnegative for $\varepsilon=1-4 /(\sqrt{4 n+1}+1)$. For each fixed $k$ in (2.10) and (2.11), set $t_{i}=h_{i i k}$. By the minimal surface equation (2.1), we have

$$
t_{1}+\cdots+t_{n}=0
$$

Step 2.1. For each fixed $k \leq m$, we prove the []$_{k}$ term in (2.10) is nonnegative. In the case with all $\lambda_{i} \geq 0$, the nonnegativity is straightforward. In the remaining worst case $\lambda_{n-1}>0>\lambda_{n}$. Without loss of generality, we assume $k=1$ for simple notation. Then we proceed as follows:

$$
\begin{gathered}
{[]_{1}=\left\{(1-\varepsilon) t_{1}^{2}+\sum_{i=2}^{m}(3-\varepsilon) t_{i}^{2}+\sum_{i=m+1}^{n-1} \frac{2 \lambda_{i}}{\lambda-\lambda_{i}} t_{i}^{2}\right\}+\frac{2 \lambda_{n}}{\lambda-\lambda_{n}} t_{n}^{2}} \\
=\left\{(1-\varepsilon) t_{1}^{2}+\sum_{i=2}^{m}(3-\varepsilon) t_{i}^{2}+\sum_{i=m+1}^{n-1} \frac{2 \lambda_{i}}{\lambda-\lambda_{i}} t_{i}^{2}\right\}+\frac{2 \lambda_{n}}{\lambda-\lambda_{n}}\left(\sum_{i=1}^{n-1} t_{i}\right)^{2} \\
\geq\left\{(1-\varepsilon) t_{1}^{2}+\sum_{i=2}^{m}(3-\varepsilon) t_{i}^{2}+\sum_{i=m+1}^{n-1} \frac{2 \lambda_{i}}{\lambda-\lambda_{i}} t_{i}^{2}\right\} . \\
{\left[1+\frac{2 \lambda_{n}}{\lambda-\lambda_{n}}\left(\frac{1}{1-\varepsilon}+\sum_{i=2}^{m} \frac{1}{3-\varepsilon}+\sum_{i=m+1}^{n-1} \frac{\lambda-\lambda_{i}}{2 \lambda_{i}}\right)\right],}
\end{gathered}
$$


where we used (2.12) and a Cauchy-Schartz inequality to reach the above inequality. We now show the second factor [] in the last term is also nonnegative:

$$
\begin{aligned}
& {\left[1+\frac{2 \lambda_{n}}{\lambda-\lambda_{n}}\left(\frac{1}{1-\varepsilon}+\sum_{i=2}^{m} \frac{1}{3-\varepsilon}+\sum_{i=m+1}^{n-1} \frac{\lambda-\lambda_{i}}{2 \lambda_{i}}\right)\right]} \\
& =\frac{2 \lambda_{n}}{\lambda-\lambda_{n}}\left(\frac{\lambda-\lambda_{n}}{2 \lambda_{n}}+\frac{1}{1-\varepsilon}+\frac{m-1}{3-\varepsilon}+\frac{\lambda-\lambda_{m+1}}{2 \lambda_{m+1}}+\cdots+\frac{\lambda-\lambda_{n-1}}{2 \lambda_{n-1}}\right) \\
& =\frac{2 \lambda_{n}}{\lambda-\lambda_{n}}\left[\frac{1}{1-\varepsilon}+\frac{m-1}{3-\varepsilon}+\frac{\lambda}{2}\left(\frac{1}{\lambda_{1}}+\cdots+\frac{1}{\lambda_{1}}\right)-\frac{n}{2}\right] \\
& =\frac{2 \lambda_{n}}{\lambda-\lambda_{n}}\left[\frac{1}{1-\varepsilon}+\frac{m-1}{3-\varepsilon}+\frac{\lambda}{2} \frac{\sigma_{n-1}}{\sigma n}-\frac{n}{2}\right] \\
& \geq \frac{2 \lambda_{n}}{\lambda-\lambda_{n}}\left(\frac{1}{1-\varepsilon}+\frac{m-1}{3-\varepsilon}-\frac{n}{2}\right) \\
& \geq 0,
\end{aligned}
$$

where we used $\lambda_{1}=\cdots=\lambda_{m}$, (2.4), and $\frac{1}{1-\varepsilon}+\frac{m-1}{3-\varepsilon}-\frac{n}{2} \leq 0$ under the assumption

$$
\varepsilon \leq 2-\frac{m}{n}-\sqrt{\left(1-\frac{m}{n}\right)^{2}+\frac{4}{n}} .
$$

Therefor []$_{1} \geq 0$.

Step 2.2. For each $k$ between $m$ and $n$, we have $\lambda_{k}>0$, the []$_{k}$ term in (2.11) satisfies

$$
\begin{aligned}
{[]_{k} } & =\sum_{i \leq m}\left(\frac{\lambda+\lambda_{k}}{\lambda-\lambda_{k}}-\varepsilon\right) t_{i}^{2} \\
& \geq \sum_{i \leq m}(1-\varepsilon) t_{i}^{2} \geq 0
\end{aligned}
$$

as long as $\varepsilon \leq 1$.

For $k=n$, the []$_{n}$ term in (2.11) becomes

$$
\begin{aligned}
{[]_{n} } & =\sum_{i \leq m}\left(\frac{\lambda+\lambda_{n}}{\lambda-\lambda_{n}}-\varepsilon\right) t_{i}^{2} \\
& \geq \sum_{i \leq m}\left(\frac{n-2}{n}-\varepsilon\right) t_{i}^{2} \geq 0
\end{aligned}
$$

where we used (2.3) and we also assumed $\varepsilon \leq \frac{n-2}{n}$.

Note that for $n-1 \geq m \geq 1$

$$
1-\frac{4}{\sqrt{4 n+1}+1} \leq 2-\frac{m}{n}-\sqrt{\left(1-\frac{m}{n}\right)^{2}+\frac{4}{n}} \leq \frac{n-2}{n},
$$


therefore we have proved (2.9) with $n-1 \geq m \geq 1$. When $m=n$, we have $\lambda_{1}=\cdots=\lambda_{n}>0$. Then from (2.10) we see in a much easier way that (2.9) holds.

The proof of Lemma 2.3 is complete.

Proposition 2.1. Let $u$ be a smooth solution to the special Lagrangian equation (1.1) with $n \geq 2$ and $\Theta \geq(n-2) \pi / 2$ on $B_{R}(0) \subset \mathbb{R}^{n}$. Set

$$
b=\ln \sqrt{1+\lambda_{\max }^{2}},
$$

where $\lambda_{\max }$ is the largest eigenvalue of Hessian $D^{2} u$, namely, $\lambda_{\max }=\lambda_{1} \geq$ $\cdots \geq \lambda_{n}$. Then $b$ satisfies the integral Jacobi inequality

$$
\int_{B_{R}}-\left\langle\nabla_{g} \varphi, \nabla_{g} b\right\rangle_{g} d v_{g} \geq \varepsilon(n) \int_{B_{R}} \varphi\left|\nabla_{g} b\right|^{2} d v_{g}
$$

for all non-negative $\varphi \in C_{0}^{\infty}\left(B_{R}\right)$, where $\varepsilon(n)=1-4 /(\sqrt{4 n+1}+1)$.

Proof. If $b(x)=b_{1}(x)$ is smooth everywhere, then the pointwise Jacobi inequality (2.9) in Lemma 2.3 with $m=1$ already implies the integral Jacobi inequality (2.13). In general, we know that $\lambda_{\max }$ is only a Lipschitz function of the entries of the Hessian $D^{2} u$. By the assumption, $D^{2} u(x)$ is smooth in $x$, thus $b=b_{1}=\ln \sqrt{1+\lambda_{\max }^{2}}$ is Lipschitz in terms of $x$.

Set $\varepsilon=\varepsilon(n)$. We first show that

$$
\triangle_{g} b \geq \varepsilon\left|\nabla_{g} b\right|^{2} \quad \text { in the viscosity sense. }
$$

Given any quadratic polynomial $Q$ touching $b$ from above at $p$. If $p$ is a smooth point of $b$, by (2.9) with $m=1$, we get

$$
\triangle_{g} Q \geq \varepsilon\left|\nabla_{g} Q\right|^{2} \quad \text { at } p .
$$

Otherwise, eigenvalue $\lambda_{1}$ is not distinct at $p$. Suppose $\lambda_{1}=\cdots=\lambda_{k}>\lambda_{k+1}$ at $p$. Then $Q$ also touches the smooth $b_{k}=\left(\ln \sqrt{1+\lambda_{1}^{2}}+\cdots+\ln \sqrt{1+\lambda_{k}^{2}}\right) / k$ from above at $p$, because

$$
b(x) \geq b_{k}(x) \text { and } b(p)=b_{k}(p) .
$$

By pointwise Jacobi inequality (2.9) with $m=k$, we still have

$$
\triangle_{g} Q \geq \varepsilon\left|\nabla_{g} Q\right|^{2} \quad \text { at } p \text {. }
$$

Next we switch to $a=e^{-\varepsilon b}$ and $a_{k}=e^{-\varepsilon b_{k}}$, the above argument leads to

$$
\triangle_{g} a \leq 0 \text { in the viscosity sense. }
$$

Relying on the definition of viscosity supersolutions, we see $a$ is $\triangle_{g}$-superharmonic in the potential sense, namely, $a \geq h$ in any regular domain $\Omega$ for $\triangle_{g^{-}}$ harmonic function $h$ with the boundary value $a$ on $\partial \Omega$ :

$$
\left\{\begin{array}{ll}
\triangle_{g} h=0 & \text { in } \Omega \\
h=a & \text { on } \partial \Omega
\end{array} .\right.
$$

By [HH, Theorem 1] (see also [Wn, p. 246]), we obtain

$$
\triangle_{g} a \leq 0 \text { in the distribution sense. }
$$


Note $a$ is Lipschitz because $b$ is. We move to the integral Jacobi inequality as follows. Take the test function $\varphi e^{\varepsilon b}$ for and nonnegative $\varphi \in C_{0}^{\infty}$, we get

$$
\begin{aligned}
0 & \geq \int_{B_{R}} \varphi e^{\varepsilon b} \triangle_{g} a d v_{g}=\int_{B_{R}}-\left\langle\nabla_{g}\left(\varphi e^{\varepsilon b}\right), \nabla_{g} a\right\rangle_{g} d v_{g} \\
& =\int_{B_{R}}\left\langle e^{\varepsilon b}\left(\nabla_{g} \varphi+\varepsilon \varphi \nabla_{g} b\right), \varepsilon e^{-\varepsilon b} \nabla_{g} b\right\rangle_{g} d v_{g} \\
& =\int_{B_{R}}\left(\varepsilon\left\langle\nabla_{g} \varphi, \nabla_{g} b\right\rangle_{g}+\varepsilon^{2} \varphi\left|\nabla_{g} b\right|_{g}^{2}\right) d v_{g} .
\end{aligned}
$$

Thus we arrive at the integral Jacobi inequality (2.13).

\section{Proof Of Theorem 1.1}

We assume that $R=2 n+1$ and $u$ is a solution on $B_{2 n+1} \subset \mathbb{R}^{n}$ for simplicity of notation. By scaling $v(x)=u\left(\frac{R}{2 n+1} x\right) /\left(\frac{R}{2 n+1}\right)^{2}$, we still get the estimate in Theorem 1.1. We consider the case $\Theta \geq(n-2) \pi / 2$. The negative phase case $\Theta \leq-(n-2) \pi / 2$ follows by symmetry.

Step 1. By the integral Jacobi inequality (2.13) in Proposition 2.1, $b$ is subharmonic in the integral sense. Then $b^{\frac{n}{n-2}}$ is also subharmonic in the integral sense on the minimal surface $\mathfrak{M}=(x, D u)$ :

$$
\begin{aligned}
& \int-\left\langle\nabla_{g} \varphi, \nabla_{g} b^{\frac{n}{n-2}}\right\rangle_{g} d v_{g} \\
& =\int-\left\langle\nabla_{g}\left(\frac{n}{n-2} b^{\frac{2}{n-2}} \varphi\right)-\frac{2 n}{(n-2)^{2}} b^{\frac{4-n}{n-2}} \varphi \nabla_{g} b, \nabla_{g} b\right\rangle_{g} d v_{g} \\
& \geq \int\left(\frac{n}{n-2} \varepsilon(n) \varphi b^{2}\left|\nabla_{g} b\right|^{2}+\frac{2 n}{(n-2)^{2}} b^{\frac{4-n}{n-2}} \varphi\left|\nabla_{g} b\right|^{2}\right) d v_{g} \geq 0
\end{aligned}
$$

for all non-negative $\varphi \in C_{0}^{\infty}$, where we approximate $b$ by smooth functions if necessary.

Applying Michael-Simon's mean value inequality [MS, Theorem 3.4] to the Lipschitz subharmonic function $b^{\frac{n}{n-2}}$, we obtain

$$
b(0) \leq C(n)\left(\int_{\mathfrak{B}_{1} \cap \mathfrak{M}} b^{\frac{n}{n-2}} d v_{g}\right)^{\frac{n-2}{n}} \leq C(n)\left(\int_{B_{1}} b^{\frac{n}{n-2}} d v_{g}\right)^{\frac{n-2}{n}},
$$

where $\mathfrak{B}_{r}$ is the ball with radius $r$ and center at $(0, D u(0))$ in $\mathbb{R}^{n} \times \mathbb{R}^{n}$, and $B_{r}$ is the ball with radius $r$ and center at 0 in $\mathbb{R}^{n}$. Choose a cut-off function $\varphi \in C_{0}^{\infty}\left(B_{2}\right)$ such that $\varphi \geq 0, \varphi=1$ on $B_{1}$, and $|D \varphi| \leq 1.1$; we then have

$$
\left(\int_{B_{1}} b^{\frac{n}{n-2}} d v_{g}\right)^{\frac{n-2}{n}} \leq\left(\int_{B_{2}} \varphi^{\frac{2 n}{n-2}} b^{\frac{n}{n-2}} d v_{g}\right)^{\frac{n-2}{n}}=\left(\int_{B_{2}}\left(\varphi b^{1 / 2}\right)^{\frac{2 n}{n-2}} d v_{g}\right)^{\frac{n-2}{n}} .
$$


Applying the Sobolev inequality on the minimal surface $\mathfrak{M}[\mathrm{MS}$, Theorem 2.1] or [A, Theorem 7.3] to $\varphi b^{1 / 2}$, which we may assume to be $C^{1}$ by approximation, we obtain

$$
\left(\int_{B_{2}}\left(\varphi b^{1 / 2}\right)^{\frac{2 n}{n-2}} d v_{g}\right)^{\frac{n-2}{n}} \leq C(n) \int_{B_{2}}\left|\nabla_{g}\left(\varphi b^{1 / 2}\right)\right|^{2} d v_{g}
$$

Decomposing the integrand as follows

$$
\begin{aligned}
\left|\nabla_{g}\left(\varphi b^{1 / 2}\right)\right|^{2} & =\left|\frac{1}{2 b^{1 / 2}} \varphi \nabla_{g} b+b^{1 / 2} \nabla_{g} \varphi\right|^{2} \leq \frac{1}{2 b} \varphi^{2}\left|\nabla_{g} b\right|^{2}+2 b\left|\nabla_{g} \varphi\right|^{2} \\
& \leq \frac{1}{\ln (4 / 3)} \varphi^{2}\left|\nabla_{g} b\right|^{2}+2 b\left|\nabla_{g} \varphi\right|^{2},
\end{aligned}
$$

where we used

$$
b \geq \ln \sqrt{1+\tan ^{2}\left(\frac{\pi}{2}-\frac{\pi}{n}\right)} \geq \ln \sqrt{4 / 3}
$$

we get

$$
\begin{aligned}
b(0) & \leq C(n) \int_{B_{2}}\left|\nabla_{g}\left(\varphi b^{1 / 2}\right)\right|^{2} d v_{g} \\
& \leq C(n)\left(\int_{B_{2}} \varphi^{2}\left|\nabla_{g} b\right|^{2} d v_{g}+\int_{B_{2}} b\left|\nabla_{g} \varphi\right|^{2} d v_{g}\right) .
\end{aligned}
$$

Step 2. By (2.13) in Proposition 2.1, $b$ satisfies the Jacobi inequality in the integral sense:

$$
\frac{1}{\varepsilon(n)} \triangle_{g} b \geq\left|\nabla_{g} b\right|^{2}
$$

Multiplying both sides by the above non-negative cut-off function $\varphi \in$ $C_{0}^{\infty}\left(B_{2}\right)$, then integrating, we obtain

$$
\begin{aligned}
\int_{B_{2}} \varphi^{2}\left|\nabla_{g} b\right|^{2} d v_{g} & \leq \frac{1}{\varepsilon(n)} \int_{B_{2}} \varphi^{2} \triangle_{g} b d v_{g} \\
& =\frac{-1}{\varepsilon(n)} \int_{B_{2}}\left\langle 2 \varphi \nabla_{g} \varphi, \nabla_{g} b\right\rangle d v_{g} \\
& \leq \frac{1}{2} \int_{B_{2}} \varphi^{2}\left|\nabla_{g} b\right|^{2} d v_{g}+\frac{2}{\varepsilon(n)^{2}} \int_{B_{2}}\left|\nabla_{g} \varphi\right|^{2} d v_{g}
\end{aligned}
$$

It follows that

$$
\int_{B_{2}} \varphi^{2}\left|\nabla_{g} b\right|^{2} d v_{g} \leq \frac{4}{\varepsilon(n)^{2}} \int_{B_{2}}\left|\nabla_{g} \varphi\right|^{2} d v_{g}
$$


So far we have reached

$$
\begin{aligned}
b(0) & \leq C(n)\left(\int_{B_{2}}\left|\nabla_{g} \varphi\right|^{2} d v_{g}+\int_{B_{2}} b\left|\nabla_{g} \varphi\right|^{2} d v_{g}\right) \\
& \leq C(n) \int_{B_{2}} b\left|\nabla_{g} \varphi\right|^{2} d v_{g} \\
& \leq C(n) \int_{B_{2}} b \sum_{i=1}^{n} \frac{1}{1+\lambda_{i}^{2}} \sqrt{\operatorname{det} g} d x,
\end{aligned}
$$

where in the second inequality, we again used $b \geq \ln \sqrt{4 / 3}$.

Step 3. Differentiating the complex identity

$$
\begin{aligned}
\ln V+\sqrt{-1} \sum_{i=1}^{n} \arctan \lambda_{i} & =\ln \prod_{i=1}^{n}\left(1+\sqrt{-1} \lambda_{i}\right) \\
& =\ln \left[\sum_{0 \leq 2 k \leq n}(-1)^{k} \sigma_{2 k}+\sqrt{-1} \sum_{1 \leq 2 k+1 \leq n}(-1)^{k} \sigma_{2 k+1}\right] .
\end{aligned}
$$

we obtain the (conformality) identity

$$
\left(\frac{1}{1+\lambda_{1}^{2}}, \cdots, \frac{1}{1+\lambda_{n}^{2}}\right) V=\left(\frac{\partial \Sigma}{\partial \lambda_{1}}, \cdots, \frac{\partial \Sigma}{\partial \lambda_{n}}\right)
$$

with $V=\sqrt{\operatorname{det} g}$ and

$$
\begin{aligned}
\Sigma & =\cos \Theta \sum_{1 \leq 2 k+1 \leq n}(-1)^{k} \sigma_{2 k+1}-\sin \Theta \sum_{0 \leq 2 k \leq n}(-1)^{k} \sigma_{2 k} \\
& =\sigma_{n-1}-\sigma_{n-3}+\cdots, \text { in particular when }|\Theta|=(n-2) \frac{\pi}{2} .
\end{aligned}
$$

Taking trace, we then get

$$
\begin{gathered}
\sum_{i=1}^{n} \frac{1}{1+\lambda_{i}^{2}} V=\sum_{i=1}^{n} \frac{\partial \Sigma}{\partial \lambda_{i}} \\
=\cos \Theta \sum_{1 \leq 2 k+1 \leq n}(-1)^{k}(n-2 k) \sigma_{2 k}-\sin \Theta \sum_{0 \leq 2 k \leq n}(-1)^{k}(n-2 k+1) \sigma_{2 k-1} \\
=c_{0}+c_{1} \sigma_{1}+\cdots+c_{n-1} \sigma_{n-1},
\end{gathered}
$$

where the coefficient $c_{i}$ depends only on $i, n$, and $\Theta$. At the critical phase $|\Theta|=(n-2) \pi / 2$, the leading term in (3.2) is $\sigma_{n-2}$

$$
\sum_{i=1}^{n} \frac{1}{1+\lambda_{i}^{2}} V=2 \sigma_{n-2}-4 \sigma_{n-4}+\cdots .
$$

In turn, (3.1) becomes

$$
b(0) \leq C(n) \int_{B_{2}} b\left(c_{0}+c_{1} \sigma_{1}+\cdots+c_{n-1} \sigma_{n-1}\right) d x .
$$


Step 4. Next we estimate the integrals $\int b \sigma_{k} d x$ for $1 \leq k \leq n-1$ inductively, using the divergence structure of $\sigma_{k}\left(D^{2} u\right)$ :

$$
\begin{aligned}
k \sigma_{k}\left(D^{2} u\right) & =\sum_{i, j=1}^{n} \frac{\partial \sigma_{k}}{\partial u_{i j}} \frac{\partial^{2} u}{\partial x_{i} \partial x_{j}}=\sum_{i, j=1}^{n} \frac{\partial}{\partial x_{i}}\left(\frac{\partial \sigma_{k}}{\partial u_{i j}} \frac{\partial u}{\partial x_{j}}\right) \\
& =\operatorname{div}\left(L_{\sigma_{k}} D u\right)
\end{aligned}
$$

where $L_{\sigma_{k}}$ denotes the matrix $\left(\frac{\partial \sigma_{k}}{\partial u_{i j}}\right)$. Let $\psi$ be a smooth cut-off function on $B_{\rho+1}$ such that $\psi=1$ on $B_{\rho}, 0 \leq \psi \leq 1$, and $|D \psi| \leq 1$.1. Noticing that $\sigma_{k}>0$ by (2.4) in Lemma 2.1 and $b>0$, we have

$$
\begin{gathered}
\int_{B_{\rho}} b \sigma_{k} d x \leq \int_{B_{\rho+1}} \psi b \sigma_{k} d x=\int_{B_{\rho+1}} \psi b \frac{1}{k} \operatorname{div}\left(L_{\sigma_{k}} D u\right) d x \\
=\frac{1}{k} \int_{B_{\rho+1}}-\left\langle b D \psi+\psi D b, L_{\sigma_{k}} D u\right\rangle d x \\
\leq C(n)\|D u\|_{L^{\infty}\left(B_{\rho+1}\right)}\left[\begin{array}{l}
\int_{B_{\rho+1}} b \sigma_{k-1} d x+ \\
\int_{B_{\rho+1}}\left[\left|\nabla_{g} b\right|^{2}+\operatorname{tr}\left(g^{i j}\right)\right] \sqrt{\operatorname{det} g} d x
\end{array}\right] .
\end{gathered}
$$

The last inequality was derived as follows. As all the above integrands are invariant under orthogonal transformations, at any point $p \in B_{\rho+1}$, we assume $D^{2} u(p)$ is diagonalized. Then $L_{\sigma_{k}}$ is also diagonal with positive entries $\partial_{\lambda_{i}} \sigma_{k}$. The positivity can be seen by applying Lemma 2.1 to all $\lambda_{1}, \cdots, \lambda_{n}$ but $\lambda_{i}$, whose corresponding phase is no less than $(n-3) \pi / 2$. Thus $0<\partial_{\lambda_{i}} \sigma_{k}<(n-k+1) \sigma_{k-1}$. Now we have

$$
\begin{aligned}
& \left|\left\langle b D \psi+\psi D b, L_{\sigma_{k}} D u\right\rangle\right| \stackrel{p}{\leq} \sum_{i=1}^{n}\left(b\left|D_{i} \psi\right|+\psi\left|D_{i} b\right|\right) \partial_{\lambda_{i}} \sigma_{k}\left|D_{i} u\right| \\
& \stackrel{p}{\leq} C(n)|D u(p)|\left(b \sigma_{k-1}+\sum_{i=1}^{n}\left|D_{i} b\right| \partial_{\lambda_{i}} \sigma_{k}\right) .
\end{aligned}
$$

Recall $k \leq n-1$, then $\partial_{\lambda_{i}} \sigma_{k}$ only consists of multiples of at most $(n-2)$ eigenvalues without $\lambda_{i}$. "Twist" multiplying the two $g^{\curvearrowright \odot}$ terms involving the missed $\lambda_{i}$ and the other eigenvalue, we obtain

$$
\begin{aligned}
& \left|D_{i} b\right| \partial_{\lambda_{i}} \sigma_{k} \stackrel{p}{\leq}\left|D_{i} b\right| \partial_{\lambda_{i}} \sigma_{k}\left(\left|\lambda_{1}\right|, \cdots,\left|\lambda_{n}\right|\right) \\
& \quad \stackrel{p}{\leq} C(n) \sum_{\alpha \neq i}\left(\frac{\left|D_{i} b\right|^{2}}{1+\lambda_{i}^{2}}+\frac{1}{1+\lambda_{\alpha}^{2}}\right) \sqrt{\left(1+\lambda_{1}^{2}\right) \cdots\left(1+\lambda_{n}^{2}\right)} .
\end{aligned}
$$

Summing up, we get

$$
\begin{aligned}
& \sum_{i=1}^{n}\left|D_{i} b\right| \partial_{\lambda_{i}} \sigma_{k} \stackrel{p}{\leq} C(n) \sum_{i=1}^{n}\left(g^{i i}\left|D_{i} b\right|^{2}+g^{i i}\right) \sqrt{\operatorname{det} g} \\
& \stackrel{p}{=} C(n)\left[\left|\nabla_{g} b\right|^{2}+\operatorname{tr}\left(g^{i j}\right)\right] \sqrt{\operatorname{det} g}
\end{aligned}
$$


The inequality (3.5) has been established. To simplify the last integral in (3.5), we repeat the integral Jacobi argument in Step 2 to get

$$
\int_{B_{\rho+1}}\left|\nabla_{g} b\right|^{2} \sqrt{\operatorname{det} g} d x \leq C(n) \int_{B_{\rho+2}} \operatorname{tr}\left(g^{i j}\right) \sqrt{\operatorname{det} g} d x .
$$

Hence (3.5) becomes the following inductive inequality

$$
\int_{B_{\rho}} b \sigma_{k} d x \leq C(n)\|D u\|_{L^{\infty}\left(B_{\rho+1}\right)}\left[\int_{B_{\rho+1}} b \sigma_{k-1} d x+\int_{B_{\rho+2}} \operatorname{tr}\left(g^{i j}\right) \sqrt{\operatorname{det} g} d x\right] .
$$

Step 4.1. We iterate (3.6) to derive

$$
\begin{gathered}
\int_{B_{2}} b \sigma_{k} d x \\
\leq C(n)\left\{\begin{array}{l}
\|D u\|_{L^{\infty}\left(B_{2+k}\right)}^{k} \int_{B_{2+k}} b d x+ \\
{\left[\|D u\|_{L^{\infty}\left(B_{2+k}\right)}^{k}+\cdots+\|D u\|_{L^{\infty}\left(B_{2+k}\right)}\right] \int_{B_{2+k+1}} \operatorname{tr}\left(g^{i j}\right) \sqrt{\operatorname{det} g} d x}
\end{array}\right\} \\
\leq C(n)\left\{\begin{array}{l}
\|D u\|_{L^{\infty}\left(B_{2+k}\right)}^{k+1}+ \\
{\left[\|D u\|_{L^{\infty}\left(B_{2+k}\right)}^{k}+\|D u\|_{L^{\infty}\left(B_{2+k}\right)}\right] \int_{B_{2+k+1}} \operatorname{tr}\left(g^{i j}\right) \sqrt{\operatorname{det} g} d x}
\end{array}\right\},
\end{gathered}
$$

where for the last inequality, we used Young's inequality and

$$
\int_{B_{2+k}} b d x \leq C(n)\|D u\|_{L^{\infty}\left(B_{2+k}\right)},
$$

which follows from

$$
b=\ln \sqrt{1+\lambda_{\max }^{2}}<\lambda_{\max }<\lambda_{1}+\lambda_{2}+\cdots+\lambda_{n}=\triangle u
$$

by (2.2) in Lemma 2.1. Putting all the estimates for $b \sigma_{k} \mathrm{~s}$ in (3.4) together, we get

$b(0) \leq C(n)\left\{\begin{array}{l}\|D u\|_{L^{\infty}\left(B_{n+1}\right)}^{n}+\|D u\|_{L^{\infty}\left(B_{n+1}\right)}+ \\ {\left[\|D u\|_{L^{\infty}\left(B_{n+1}\right)}^{n-1}+\|D u\|_{L^{\infty}\left(B_{n+1}\right)}\right] \int_{B_{n+2}} \operatorname{tr}\left(g^{i j}\right) \sqrt{\operatorname{det} g} d x}\end{array}\right\}$.

Step 4.2. We bound the last integral in the above inequality. Relying on the trace conformality identity (3.2), we derive

$$
\begin{aligned}
\int_{B_{n+2}} \operatorname{tr}\left(g^{i j}\right) \sqrt{\operatorname{det} g} d x & =\int_{B_{n+2}}\left(c_{0}+c_{1} \sigma_{1}+\cdots+c_{n-1} \sigma_{n-1}\right) d x \\
& \leq C(n)\left[\|D u\|_{L^{\infty}\left(B_{2 n+1}\right)}^{n-1}+1\right]
\end{aligned}
$$

where for the last inequality, we repeated the iteration integral estimates for (3.6) in Step 4.1 with $b=1$ (now much simpler)

$$
\int_{B_{\rho}} \sigma_{k} d x \leq C(n)\|D u\|_{L^{\infty}\left(B_{\rho+1}\right)} \int_{B_{\rho+1}} \sigma_{k-1} d x
$$


Finally from the above estimates (3.8) and (3.7), we conclude that

$$
b(0) \leq C(n)\left[\|D u\|_{L^{\infty}\left(B_{2 n+1}\right)}^{2 n-2}+\|D u\|_{L^{\infty}\left(B_{2 n+1}\right)}\right]
$$

and after exponentiating

$$
\left|D^{2} u(0)\right| \leq C(n) \exp \left[C(n)\|D u\|_{L^{\infty}\left(B_{2 n+1}\right)}^{2 n-2}\right] .
$$

Note at the critical phase $\Theta=(n-2) \pi / 2$, because of (3.3), the leading term in (3.4) and (3.8) is $\sigma_{n-2}$. The iteration integral estimates in Step 4.1 and 4.2 start from $\sigma_{n-2}$. Thus we really obtain

$$
\left|D^{2} u(0)\right| \leq C(n) \exp \left[C(n)\|D u\|_{L^{\infty}\left(B_{2 n}\right)}^{2 n-4}\right] .
$$

The proof of Theorem 1.1 is complete.

\section{REFERENCES}

[A] Allard, William K., On the first variation of a varifold. Ann. of Math. (2) 95 (1972), 417-491.

[BC] Bao, Jiguang and Chen, Jingyi, Optimal regularity for convex strong solutions of special Lagrangian equations in dimension 3. Indiana Univ. Math. J. 52 (2003), 1231-1249.

[BCGJ] Bao, Jiguang, Chen, Jingyi, Guan, Bo, and Ji, Min, Amer. J. Math. 125 (2003), 301-316.

[BDM] Bombieri, Enrico, De Giorgi, Ennio and Miranda, Mario, Una maggiorazione a priori relativa alle ipersuperfici minimali non parametriche. Arch. Rational Mech. Anal. 32 (1969), 255-267.

[CWY] Chen, Jingyi, Warren, Micah, and Yuan, Yu, A priori estimate for convex solutions to special Lagrangian Equations and its application. Comm. Pure Appl. Math. 62 (2009), no. 4, 583-595.

[CW] Chou, Kai-Seng and Wang, Xu-Jia, A variational theory of the Hessian equation. Comm. Pure Appl. Math. 54 (2001), 1029-1064.

[F] Finn, Robert, New estimates for equations of minimal surface type, Arch. Rational Mech. Anal. 14 (1963), 337-375.

[HL] Harvey, Reese and Lawson, H. Blaine. Jr., Calibrated geometry. Acta Math. 148 (1982), 47-157.

[H] Heinz, Erhard, On elliptic Monge-Ampère equations and Weyl's embedding problem. J. Analyse Math. 7 (1959) 1-52.

[HH] Hervé, Rose-Marie and Hervé, Michel, Les fonctions surharmoniques dans l'axiomatique de M. Brelot associées àun opéateur elliptique dégénéré. (French) Ann. Inst. Fourier (Grenoble) 22 (1972), no. 2, 131-145.

[J] Jögens, Konrad, Üer die Löungen der Differentialgleichung $r t-s^{2}=1$. (German) Math. Ann. 127 (1954), 130-134.

[K] N. Korevaar, An easy proof of the interior gradient bound for solutions to the prescribed mean curvature equation. Nonlinear functional analysis and its applications, Part 2 (Berkeley, Calif., 1983), 81-89, Proc. Sympos. Pure Math., 45, Part 2, Amer. Math. Soc., Providence, RI, 1986.

[MS] Michael, James H. and Simon, Leon M., Sobolev and mean-value inequalities on generalized submanifolds of $\mathbb{R}^{n}$. Comm. Pure Appl. Math. 26 (1973), 361-379.

[NV] Nadirashvili, Nicolai and Vlăduţ, Serge, Singular solution to Special Lagrangian Equations. Ann. Inst. H. Poincaré Anal. Non Linéaire 27 (2010), no. 5, 1179-1188. 
[P1] Pogorelov, Aleksei Vasil'evich, Monge-Ampère Equations of Elliptic Type. Translated from the first Russian edition by Leo F. Boron with the assistance of Albert L. Rabenstein and Richard C. Bollinger P. Noordhoff, Ltd., Groningen, 1964

[P2] Pogorelov, Aleksei Vasil'evich, The Minkowski Multidimensional Problem. Translated from the Russian by Vladimir Oliker. Introduction by Louis Nirenberg. Scripta Series in Mathematics. V. H. Winston \& Sons, Washington, D.C.; Halsted Press [John Wiley \& Sons], New York-Toronto-London, 1978.

[T1] Trudinger, Neil S. A new proof of the interior gradient bound for the minimal surface equation in $n$ dimensions. Proc. Nat. Acad. Sci. U.S.A. 69 (1972), 821823.

[T2] Trudinger, Neil S. Regularity of solutions of fully nonlinear elliptic equations. Boll. U.M.I. (6) 3-A (1984), 421-430.

[U1] Urbas, John I. E., On the existence of nonclassical solutions for two classes of fully nonlinear elliptic equations. Indiana Univ. Math. J. 39 (1990), no. 2, 355-382.

[U2] Urbas, John, Some interior regularity results for solutions of Hessian equations. Calc. Var. Partial Differential Equations 11 (2000), 1-31.

[U3] Urbas, John, An interior second derivative bound for solutions of Hessian equations. Calc. Var. Partial Differential Equations 12 (2001), 417-431.

[WdY] Wang, Dake and Yuan, Yu, Singular solutions to special Lagrangian equations with subcritical phases and minimal surface systems. Amer. J. Math., to appear.

[WY1] Warren, Micah and Yuan, Yu, A Liouville type theorem for special Lagrangian Equations with constraints. Comm. Partial Differential Equations 33 (2008), 922932.

[WY2] Warren, Micah and Yuan, Yu, Hessian estimates for the sigma-2 equation in dimension three. Comm. Pure Appl. Math. 62 (2009), no. 3, 305-321.

[WY3] Warren, Micah and Yuan, Yu, Explicit gradient estimates for minimal Lagrangian surfaces of dimension two. Math. Z. 262 (2009), no. 4, 867-879.

[WY4] Warren, Micah and Yuan, Yu, Hessian and gradient estimates for three dimensional special Lagrangian Equations with large phase. Amer. J. Math. 132 (2010), no. 3, 751-770.

[Wn] Watson, Neil A., Generalizations of the spherical mean convexity theorem on subharmonic functions. Ann. Acad. Sci. Fenn. Ser. A I Math. 17 (1992), no. 2, 241-255.

[Y1] Yuan, Yu, A Bernstein problem for special Lagrangian equations. Invent. Math. 150 (2002), 117-125.

[Y2] Yuan, Yu, Global solutions to special Lagrangian equations. Proc. Amer. Math. Soc. 134 (2006), no. 5, 1355-1358.

Department of Mathematics, Box 354350, University of Washington, SeatTLE, WA 98195

E-mail address: dkpool@math.washington.edu, yuan@math.washington.edu 


\title{
HESSIAN ESTIMATES FOR SPECIAL LAGRANGIAN EQUATIONS WITH CRITICAL AND SUPERCRITICAL PHASES IN GENERAL DIMENSIONS
}

\author{
DAKE WANG AND YU YUAN
}

\begin{abstract}
We derive a priori interior Hessian estimates for special Lagrangian equation with critical and supercritical phases in general higher dimensions. Our unified approach leads to sharper estimates even for the previously known three dimensional and convex solution cases.
\end{abstract}

\section{INTRODUCTION}

In this paper, we complete a priori interior Hessian estimates for the special Lagrangian equation

$$
\sum_{i=1}^{n} \arctan \lambda_{i}=\Theta
$$

with critical and supercritical phases $|\Theta| \geq(n-2) \pi / 2$ in all dimensions $n \geq 3$, where $\lambda=\left(\lambda_{1}, \cdots, \lambda_{n}\right)$ are the eigenvalues of the Hessian $D^{2} u$. For solutions to (1.1) with $|\Theta| \geq(n-2) \pi / 2$ in dimension two and three, and also convex solutions to (1.1) in all dimensions, Hessian estimates have been obtained in [WY2,3,4] and [CWY].

Equation (1.1) originates in the special Lagrangian geometry by HarveyLawson [HL]. The Lagrangian graph $(x, D u(x)) \subset \mathbb{R}^{n} \times \mathbb{R}^{n}$ is called special when the argument of the complex number $\left(1+\sqrt{-1} \lambda_{1}\right) \cdots\left(1+\sqrt{-1} \lambda_{n}\right)$ or the phase is constant $\Theta$, and it is special if and only if $(x, D u(x))$ is a (volume minimizing) minimal surface in $\mathbb{R}^{n} \times \mathbb{R}^{n}$ [HL, Theorem 2.3, Proposition 2.17]. The phase $(n-2) \pi / 2$ is called critical because the level set $\left\{\lambda \in \mathbb{R}^{n} \mid \lambda\right.$ satisfying (1.1) $\}$ is convex only when $|\Theta| \geq(n-2) \pi / 2[\mathrm{Y} 2$, Lemma 2.1]. The algebraic form of (1.1) is

$$
\cos \Theta \sum_{1 \leq 2 k+1 \leq n}(-1)^{k} \sigma_{2 k+1}-\sin \Theta \sum_{0 \leq 2 k \leq n}(-1)^{k} \sigma_{2 k}=0,
$$

where $\sigma_{k}$ s are the elementary symmetric functions of the Hessian $D^{2} u$.

We state our main result in the following.

Date: October 20, 2018.

Both authors are partially supported by an NSF grant. 
Theorem 1.1. Let $u$ be a smooth solution to (1.1) with $|\Theta| \geq(n-2) \pi / 2$ and $n \geq 3$ on $B_{R}(0) \subset \mathbb{R}^{n}$. Then we have

$$
\left|D^{2} u(0)\right| \leq C(n) \exp \left[C(n) \max _{B_{R}(0)}|D u|^{2 n-2} / R^{2 n-2}\right] ;
$$

and when $|\Theta|=(n-2) \pi / 2$, we also have

$$
\left|D^{2} u(0)\right| \leq C(n) \exp \left[C(n) \max _{B_{R}(0)}|D u|^{2 n-4} / R^{2 n-4}\right] .
$$

Relying on our previous gradient estimates for (1.1) with $|\Theta| \geq(n-2) \pi / 2$ in [WY4]

$$
\max _{B_{R}(0)}|D u| \leq C(n)\left[\underset{B_{2 R}(0)}{\operatorname{osc}} \frac{u}{R}+1\right],
$$

we can bound $D^{2} u$ in terms of the solution $u$ in $B_{2 R}(0)$.

Singular (viscosity) solutions to (1.1) with subcritical phases $|\Theta|<(n-2) \pi / 2$ and $n \geq 3$ constructed by Nadirashvili-Vlădut [NV] and the authors [WdY] show that the critical and supercritical phase condition in Theorem 1.1 is necessary.

One application of the above estimates is the regularity (analyticity) of the $C^{0}$ viscosity solutions to (1.1) with $|\Theta| \geq(n-2) \pi / 2$. In particular, the solutions of the Dirichlet problem with continuous boundary data to (1.1) with convex condition $|\Theta| \geq(n-2) \pi / 2$ enjoy interior regularity. In contrast, the Hessian estimates, then the interior regularity for solutions to (1.1) with $|\Theta|=\left[\frac{n-1}{2}\right] \pi$ in [CNS] by Caffarelli-Nirenberg-Spruck was derived under the $C^{4}$ smoothness assumption on the boundary data.

Another quick consequence is a Liouville type result for global solutions with quadratic growth to (1.1) with $|\Theta|=(n-2) \pi / 2$, namely any such a solution must be quadratic (cf. [Y1], [Y2] where other Liouville type results for convex solutions to (1.1) and Bernstein type results for global solutions to (1.1) with supercritical phase $|\Theta|>(n-2) \pi / 2$ were obtained).

In the 1950's, Heinz $[\mathrm{H}]$ derived a Hessian bound for the two dimensional Monge-Ampère type equation including (1.1) with $n=2$; see also Pogorelov [P1] for Hessian estimates for these equations including (1.1) with $|\Theta|>\pi / 2$ and $n=2$. In the 1970's Pogorelov [P2] constructed his famous counterexamples, namely irregular solutions to three dimensional MongeAmpère equations $\sigma_{3}\left(D^{2} u\right)=\operatorname{det}\left(D^{2} u\right)=1$; those irregular solutions also serve as counterexamples for cubic and higher order symmetric $\sigma_{k}$ equations (cf. [U1]). In passing, we also mention Hessian estimates for solutions with certain strict convexity constraints to Monge-Ampère equations and $\sigma_{k}$ equation $(k \geq 2)$ by Pogorelov [P2] and Chou-Wang [CW] respectively using the Pogorelov technique. Trudinger [T2] and Urbas [U2][U3], also Bao-Chen $[\mathrm{BC}]$ obtained (pointwise) Hessian estimates in terms of certain integrals of the Hessian, for $\sigma_{k}$ equations and special Lagrangian equation (1.1) with 
$n=3, \Theta=\pi$ respectively. Pointwise Hessian estimates for strictly convex solutions to quotient equations $\sigma_{n} / \sigma_{k}$ were derived in terms of certain integrals of the Hessian by Bao-Chen-Guan-Ji [BCGJ].

Our strategies for the Hessian estimates go as follows. We bound the subharmonic function of the Hessian $b=\ln \sqrt{1+\lambda_{\max }^{2}}$ by its integral on the minimal surface using Michael-Simon's mean value inequality [MS]. Applying certain Sobolev inequalities, we estimate the integral of $b$ by the integral of its gradient. The decisive choice $b$ satisfies a Jacobi inequality: its Laplacian bounds its gradient; in turn, the integral of the gradient $b$ is bounded by a weighted volume of the minimal Lagrangian graph. By a conformality identity, the weighted volume element is in fact the trace of the linearized operator of the special Lagrangian equation in algebraic form, which is a linear combination of the elementary symmetric functions of the Hessian. Taking advantage of the divergence structure of those functions, we bound the weighted volume in terms of the height of special Lagrangian graph, or the gradient of the solution.

However, there are two major difficulties in the execution for general dimension. The first one is to justify the nonlinear Jacobi inequality in the integral sense for the Lipschitz only function $b$, which was only achieved in dimension three by involved arguments [WY2]. The second one is to find, in the critical phase case, a relative isoperimetric inequality or equivalent Sobolev inequality for functions without compact support, which was circumvented only in dimension three thanks to the linear dependence on the Hessian for the linearized operator of now equivalent equation $\sigma_{2}=1$ [WY2]. We overcome the first one by observing that the Jacobi inequality and its equivalent linear formulation hold in the viscosity sense, consequently in the potential sense. By Hervé-Hervé [HH, Theorem 1] (see also Watson [Wn, p. 246]), the linear inequality holds in the integral sense, in turn, so does the needed Jacobi inequality. Conceptually it is natural this way. For details, see the proof of Proposition 2.1. To deal with the second difficulty, we instead apply the Sobolev inequality for functions with compact supports, but use a "twist-multiplication" trick to contain the terms involving derivatives of the cut-off functions (Step 4 in Section 3). This trick enables us to have a unified approach (for both the critical and supercritical cases) in all dimensions $n \geq 3$. Even in the known three dimensional [WY2,4] and convex cases [CWY], the simpler unified argument leads to sharper Hessian estimates.

Our unified arguments does not work for (1.1) with $\Theta=0$ and $n=2$, as the Jacobi inequality fails (only) for harmonic functions. Elementary methods in [WY3] led to the sharp Hessian estimates in dimension two. (The sharp Hessian estimates in terms of the linear exponential dependence on the gradients, can be seen by the corresponding solutions to the MongeAmpère equation or (1.1) with $\Theta=\pi / 2$ and $n=2$, converted from Finn's minimal surface $[\mathrm{F}$, p. 355] via Heinz transformation [J, p. 133].) 
As one can see that, not only our Hessian-slope estimates for "gradient" minimal graphs are analogous to the gradient-slope estimates for the codimension one minimal graphs, but also our arguments resemble the original integral proof by Bombieri-De Giorgi-Miranda [BDM] and the simplified one by Trudinger [T1] for the latter classical result. When one tries to adapt the later Korevaar pointwise technique $[\mathrm{K}]$, certain extra structure or assumption has to be used, as in [WY1]. Otherwise, an adaptation of the technique alone would lead to Hessian estimates for the Monge-Ampère equations, to which the Jacobi inequality is available. But this is inconsistent with Pogorelov's singular solutions [P2].

Notation. First $\partial_{i}=\frac{\partial}{\partial x_{i}}, \partial_{i j}=\frac{\partial^{2}}{\partial x_{i} \partial x_{j}}, u_{i}=\partial_{i} u=D_{i} u, u_{j i}=\partial_{i j} u$ etc., but $\lambda_{1}, \cdots, \lambda_{n}$ and $b_{k}=\left(\ln \sqrt{1+\lambda_{1}^{2}}+\cdots+\ln \sqrt{1+\lambda_{k}^{2}}\right) / k$ do not represent the partial derivatives. Also

$$
\sigma_{k}\left(\lambda_{1}, \cdots, \lambda_{n}\right)=\sum_{1 \leq i_{1}<\cdots<i_{k} \leq n} \lambda_{i_{1}} \cdots \lambda_{i_{k}} .
$$

Further, $h_{i j k}$ will denote (the second fundamental form)

$$
h_{i j k}=\frac{1}{\sqrt{1+\lambda_{i}^{2}}} \frac{1}{\sqrt{1+\lambda_{j}^{2}}} \frac{1}{\sqrt{1+\lambda_{k}^{2}}} u_{i j k} .
$$

when $D^{2} u$ is diagonalized. Finally $C(n)$ will denote various constants depending only on dimension $n$.

\section{Preliminary inequalities}

Taking the gradient of both sides of the special Lagrangian equation (1.1), we have

$$
\sum_{i, j=1}^{n} g^{i j} \partial_{i j}(x, D u(x))=0,
$$

where $\left(g^{i j}\right)$ is the inverse of the induced metric $g=\left(g_{i j}\right)=I+D^{2} u D^{2} u$ on the surface $(x, D u(x)) \subset \mathbb{R}^{n} \times \mathbb{R}^{n}$. Simple geometric manipulation of (2.1) yields the usual form of the minimal surface equation

$$
\triangle_{g}(x, D u(x))=0 \text {, }
$$

where the Laplace-Beltrami operator of the metric $g$ is given by

$$
\triangle_{g}=\frac{1}{\sqrt{\operatorname{det} g}} \sum_{i, j=1}^{n} \partial_{i}\left(\sqrt{\operatorname{det} g} g^{i j} \partial_{j}\right) .
$$

Because we are using harmonic coordinates $\triangle_{g} x=0$, we see that $\triangle_{g}$ also equals the linearized operator of the special Lagrangian equation (1.1) at $u$,

$$
\triangle_{g}=\sum_{i, j=1}^{n} g^{i j} \partial_{i j}
$$


The volume form, gradient and inner product with respect to the metric $g$ are

$$
\begin{aligned}
d v_{g} & =\sqrt{\operatorname{det} g} d x \\
\nabla_{g} v & =\left(\sum_{k=1}^{n} g^{1 k} v_{k}, \cdots, \sum_{k=1}^{n} g^{n k} v_{k}\right), \\
\left\langle\nabla_{g} v, \nabla_{g} w\right\rangle_{g} & =\sum_{i, j=1}^{n} g^{i j} v_{i} w_{j}, \text { in particular }\left|\nabla_{g} v\right|^{2}=\left\langle\nabla_{g} v, \nabla_{g} v\right\rangle_{g} .
\end{aligned}
$$

We begin with some algebraic and trigonometric inequalities needed in this paper.

Lemma 2.1. Suppose the ordered real numbers $\lambda_{1} \geq \lambda_{2} \geq \cdots \geq \lambda_{n}$ satisfy (1.1) with $\Theta \geq(n-2) \pi / 2$ and $n \geq 2$. Then we have

$$
\begin{gathered}
\lambda_{1} \geq \lambda_{2} \geq \cdots \geq \lambda_{n-1}>0 \text { and } \lambda_{n-1} \geq\left|\lambda_{n}\right|, \\
\lambda_{1}+(n-1) \lambda_{n} \geq 0, \\
\sigma_{k}\left(\lambda_{1}, \cdots, \lambda_{n}\right) \geq 0 \text { for all } 1 \leq k \leq n-1 .
\end{gathered}
$$

Proof. Set $\theta_{i}=\arctan \lambda_{i}$. Property (2.2) follows from the inequalities

$$
\theta_{n-1}+\theta_{n} \geq(n-2) \pi / 2-\left(\theta_{1}+\cdots+\theta_{n-2}\right) \geq 0 .
$$

We only need to check property (2.3) when $\lambda_{n}<0$ or $\theta_{n}<0$. We know

$$
\frac{\pi}{2}>\frac{\pi}{2}+\theta_{n} \geq\left(\frac{\pi}{2}-\theta_{1}\right)+\cdots+\left(\frac{\pi}{2}-\theta_{n-1}\right)>0 .
$$

It follows that

$$
\begin{aligned}
-\frac{1}{\lambda_{n}} & =\tan \left(\frac{\pi}{2}+\theta_{n}\right) \\
& \geq \tan \left(\frac{\pi}{2}-\theta_{1}\right)+\cdots+\tan \left(\frac{\pi}{2}-\theta_{n-1}\right)=\frac{1}{\lambda_{1}}+\cdots+\frac{1}{\lambda_{n-1}} \\
& \geq(n-1) \frac{1}{\lambda_{1}} .
\end{aligned}
$$

Then we get (2.3).

Next we prove property (2.4) with $k=n-1$. We only need to deal with the case $\lambda_{n}<0$. From (2.5), we have

$$
0 \geq \frac{1}{\lambda_{1}}+\cdots+\frac{1}{\lambda_{n-1}}+\frac{1}{\lambda_{n}}=\frac{\sigma_{n-1}\left(\lambda_{1}, \cdots, \lambda_{n}\right)}{\left(\lambda_{1} \cdots \lambda_{n-1}\right) \lambda_{n}} .
$$

Using $\lambda_{1} \geq \lambda_{2} \geq \cdots \geq \lambda_{n-1}>0>\lambda_{n}$, we get $\sigma_{n-1}\left(\lambda_{1}, \cdots, \lambda_{n}\right) \geq 0$.

Finally we prove the whole property (2.4) inductively. Property (2.4) with $n=2$ is obvious (or by the above). Assume property (2.4) with $n=m$ is true, that is

$$
\sigma_{j}\left(\lambda_{1}, \cdots, \lambda_{m}\right) \geq 0 \text { for } 1 \leq j \leq m-1,
$$

provided $\arctan \lambda_{1}+\cdots+\arctan \lambda_{m} \geq(m-2) \pi / 2$. 
Let us prove (2.4) with $n=m+1$ for

$$
\arctan \lambda_{1}+\cdots+\arctan \lambda_{m+1} \geq(m-1) \pi / 2 \text {. }
$$

By the proved property (2.4) with $k=n-1=m$, we get $\sigma_{m}\left(\lambda_{1}, \cdots, \lambda_{m+1}\right) \geq$ 0 . We only need to verify the other $\sigma$ inequalities when the smallest number is negative, say $\lambda_{1} \geq \lambda_{2} \geq \cdots \geq \lambda_{m}>0>\lambda_{m+1}$. (By (2.2), only the smallest $\lambda_{m+1}$ can be negative.) We have

$$
\sigma_{m-1}\left(\lambda_{1}, \cdots, \lambda_{m+1}\right)=\sigma_{m-1}\left(\lambda_{2}, \cdots, \lambda_{m+1}\right)+\lambda_{1} \sigma_{m-2}\left(\lambda_{2}, \cdots, \lambda_{m+1}\right) .
$$

From (2.6), we infer

$$
\arctan \lambda_{2}+\cdots+\arctan \lambda_{m+1} \geq(m-2) \pi / 2 .
$$

By the induction assumption, we should have

$$
\sigma_{m-1}\left(\lambda_{2}, \cdots, \lambda_{m+1}\right) \geq 0 \text { and } \sigma_{m-2}\left(\lambda_{2}, \cdots, \lambda_{m+1}\right) \geq 0 .
$$

Thus we obtain $\sigma_{m-1}\left(\lambda_{1}, \cdots, \lambda_{m+1}\right) \geq 0$. Similarly we prove $\sigma_{i}\left(\lambda_{1}, \cdots, \lambda_{m+1}\right) \geq$ 0 for $1 \leq i \leq m-2$. Therefore property (2.4) holds for all $n \geq 2$. This completes the proof of Lemma 2.1.

Lemma 2.2. Let $u$ be a smooth solution to (1.1). Suppose that the Hessian $D^{2} u$ is diagonalized and the eigenvalue $\lambda_{\gamma}$ is distinct from all other eigenvalues of $D^{2} u$ at point $p$. Then we have at $p$

$$
\left|\nabla_{g} \ln \sqrt{1+\lambda_{\gamma}^{2}}\right|^{2}=\sum_{k=1}^{n} \lambda_{\gamma}^{2} h_{\gamma \gamma k}^{2}
$$

and

$$
\begin{aligned}
& \triangle_{g} \ln \sqrt{1+\lambda_{\gamma}^{2}}= \\
& \left(1+\lambda_{\gamma}^{2}\right) h_{\gamma \gamma \gamma}^{2}+\sum_{k \neq \gamma}\left(\frac{2 \lambda_{\gamma}}{\lambda_{\gamma}-\lambda_{k}}+\frac{2 \lambda_{\gamma}^{2} \lambda_{k}}{\lambda_{\gamma}-\lambda_{k}}\right) h_{k k \gamma}^{2} \\
& +\sum_{k \neq \gamma}\left[1+\frac{2 \lambda_{\gamma}}{\lambda_{\gamma}-\lambda_{k}}+\frac{\lambda_{\gamma}^{2}\left(\lambda_{\gamma}+\lambda_{k}\right)}{\lambda_{\gamma}-\lambda_{k}}\right] h_{\gamma \gamma k}^{2} \\
& +\sum_{\substack{k>j \\
k, j \neq \gamma}} 2 \lambda_{\gamma}\left[\frac{1+\lambda_{k}^{2}}{\lambda_{\gamma}-\lambda_{k}}+\frac{1+\lambda_{j}^{2}}{\lambda_{\gamma}-\lambda_{j}}+\left(\lambda_{j}+\lambda_{k}\right)\right] h_{k j \gamma}^{2} .
\end{aligned}
$$

Proof. The calculation was done in Lemma 2.1 of [WY2].

Lemma 2.3. Let $u$ be a smooth solution to (1.1) with $\Theta \geq(n-2) \frac{\pi}{2}$. Suppose that the ordered eigenvalues $\lambda_{1} \geq \lambda_{2} \geq \cdots \geq \lambda_{n}$ of the Hessian $D^{2} u$ satisfy $\lambda_{1}=\cdots=\lambda_{m}>\lambda_{m+1}$ at point $p$. Then the function $b_{m}=\frac{1}{m} \sum_{i=1}^{m} \ln \sqrt{1+\lambda_{i}^{2}}$ is smooth near $p$ and satisfies at $p$

$$
\triangle_{g} b_{m} \geq\left(1-\frac{4}{\sqrt{4 n+1}+1}\right)\left|\nabla_{g} b_{m}\right|^{2} .
$$


Proof. Step 1. The function $b_{m}$ is symmetric in $\lambda_{1}, \cdots, \lambda_{m}$. Thus for $m<n$, $b_{m}$ is smooth when $\lambda_{m}>\lambda_{m+1}$, in particular near $p$, at which $\lambda_{1}=\cdots=$ $\lambda_{m}>\lambda_{m+1}$. For $m=n, b_{n}$ is certainly smooth everywhere.

We again assume that Hessian $D^{2} u$ is diagonalized at point $p$. Let us also first assume the first $m$ eigenvalues $\lambda_{1}, \cdots, \lambda_{m}$ are distinct. Using (2.8) in Lemma 2.2, we calculate $\triangle_{g} b_{m}$; after grouping those terms $h_{\diamond \diamond \diamond}, h_{\odot \odot}$ and $h_{\odot} \diamond$ in the summation, we obtain

$$
\begin{aligned}
& m \triangle_{g} b_{m}=\sum_{\gamma=1}^{m} \triangle_{g} \ln \sqrt{1+\lambda_{\gamma}^{2}} \stackrel{p}{=} \\
& \sum_{k \leq m}\left(1+\lambda_{k}^{2}\right) h_{k k k}^{2}+\left(\sum_{i<k \leq m}+\sum_{k<i \leq m}\right)\left(3+\lambda_{i}^{2}+2 \lambda_{i} \lambda_{k}\right) h_{i i k}^{2}+\sum_{k \leq m<i} \frac{2 \lambda_{k}\left(1+\lambda_{k} \lambda_{i}\right)}{\lambda_{k}-\lambda_{i}} h_{i i k}^{2} \\
& +\sum_{i \leq m<k} \frac{3 \lambda_{i}-\lambda_{k}+\lambda_{i}^{2}\left(\lambda_{i}+\lambda_{k}\right)}{\lambda_{i}-\lambda_{k}} h_{i i k}^{2}+ \\
& \left\{\begin{array}{l}
2 \sum_{i<j<k \leq m}\left(3+\lambda_{i} \lambda_{j}+\lambda_{j} \lambda_{k}+\lambda_{k} \lambda_{i}\right) h_{i j k}^{2}+ \\
2 \sum_{i<j \leq m<k}\left(1+\lambda_{i} \lambda_{j}+\lambda_{j} \lambda_{k}+\lambda_{k} \lambda_{i}+\lambda_{i} \frac{1+\lambda_{k}^{2}}{\lambda_{i}-\lambda_{k}}+\lambda_{j} \frac{1+\lambda_{k}^{2}}{\lambda_{j}-\lambda_{k}}\right) h_{i j k}^{2}+. \\
2 \sum_{i \leq m<j<k} \lambda_{i}\left[\lambda_{j}+\lambda_{k}+\frac{1+\lambda_{j}^{2}}{\lambda_{i}-\lambda_{j}}+\frac{1+\lambda_{k}^{2}}{\lambda_{j}-\lambda_{k}}\right] h_{i j k}^{2}
\end{array}\right.
\end{aligned}
$$

Now as a function of the matrices (then composed with smooth matrix function $D^{2} u$ of $\left.x\right), b_{m}$ is $C^{2}$ at $D^{2} u(p)$ with eigenvalues satisfying $\lambda=$ $\lambda_{1}=\cdots=\lambda_{m}>\lambda_{m+1}$. Note that $D^{2} u(p)$ can be approximated by matrices with distinct eigenvalues. Therefore the above expression for $\triangle_{g} b_{m}$ at $p$ still holds and simplifies to

$$
\begin{aligned}
& m \triangle_{g} b_{m} \underline{\underline{p}} \\
& \sum_{k \leq m}\left(1+\lambda^{2}\right) h_{k k k}^{2}+\left(\sum_{i<k \leq m}+\sum_{k<i \leq m}\right)\left(3+3 \lambda^{2}\right) h_{i i k}^{2}+\sum_{k \leq m<i} \frac{2 \lambda\left(1+\lambda \lambda_{i}\right)}{\lambda-\lambda_{i}} h_{i i k}^{2}+ \\
& \sum_{i \leq m<k} \frac{3 \lambda-\lambda_{k}+\lambda^{2}\left(\lambda+\lambda_{k}\right)}{\lambda-\lambda_{k}} h_{i i k}^{2}+ \\
& \left\{\begin{array}{l}
2 \sum_{i<j<k \leq m}\left(3+3 \lambda^{2}\right) h_{i j k}^{2}+ \\
2 \sum_{i<j \leq m<k}\left[1+\frac{2 \lambda}{\lambda-\lambda_{k}}+\frac{\lambda^{2}\left(\lambda+\lambda_{k}\right)}{\lambda-\lambda_{k}}\right] h_{i j k}^{2}+ \\
2 \sum_{i \leq m<j<k} \lambda\left(\lambda_{j}+\lambda_{k}+\frac{1+\lambda_{j}^{2}}{\lambda-\lambda_{j}}+\frac{1+\lambda_{k}^{2}}{\lambda-\lambda_{k}}\right) h_{i j k}^{2} \\
\geq \sum_{k \leq m} \lambda^{2} h_{k k k}^{2}+\left(\sum_{i<k \leq m}+\sum_{k<i \leq m}\right) 3 \lambda^{2} h_{i i k}^{2}+\sum_{k \leq m<i} \frac{2 \lambda^{2} \lambda_{i}}{\lambda-\lambda_{i}} h_{i i k}^{2}+ \\
\sum_{i \leq m<k} \frac{\lambda^{2}\left(\lambda+\lambda_{k}\right)}{\lambda-\lambda_{k}} h_{i i k}^{2},
\end{array}\right.
\end{aligned}
$$

where we used (2.2) of Lemma 2.1 in the inequality. 
Similarly by (2.7) in Lemma 2.2 and the $C^{1}$ continuity of $b_{m}$ as a function of matrices at $D^{2} u(p)$, we obtain

$$
\left|\nabla_{g} b_{m}\right|^{2} \stackrel{p}{=} \frac{1}{m^{2}} \sum_{1 \leq k \leq n} \lambda^{2}\left(\sum_{i \leq m} h_{i i k}\right)^{2} \leq \frac{\lambda^{2}}{m} \sum_{1 \leq k \leq n}\left(\sum_{i \leq m} h_{i i k}^{2}\right)
$$

From the above two inequalities, it follows that

$$
\begin{gathered}
m\left(\triangle_{g} b_{m}-\varepsilon\left|\nabla_{g} b_{m}\right|^{2}\right) \geq \\
\lambda^{2}\left[\begin{array}{c}
\sum_{k \leq m}(1-\varepsilon) h_{k k k}^{2}+\left(\sum_{i<k \leq m}+\sum_{2 \lambda}+\sum_{k<i \leq m}\right)(3-\varepsilon) h_{i i k}^{2} \\
+\sum_{k \leq m<i} \frac{\lambda-\lambda_{i}}{2} h_{i i k}
\end{array}\right]+ \\
\lambda^{2}\left[\sum_{i \leq m<k}\left(\frac{\lambda+\lambda_{k}}{\lambda-\lambda_{k}}-\varepsilon\right) h_{i i k}^{2}\right]
\end{gathered}
$$

with $\varepsilon$ to be fixed.

Step 2. We show (2.10) and (2.11) in the above inequality are nonnegative for $\varepsilon=1-4 /(\sqrt{4 n+1}+1)$. For each fixed $k$ in (2.10) and (2.11), set $t_{i}=h_{i i k}$. By the minimal surface equation (2.1), we have

$$
t_{1}+\cdots+t_{n}=0
$$

Step 2.1. For each fixed $k \leq m$, we prove the []$_{k}$ term in (2.10) is nonnegative. In the case with all $\lambda_{i} \geq 0$, the nonnegativity is straightforward. In the remaining worst case $\lambda_{n-1}>0>\lambda_{n}$. Without loss of generality, we assume $k=1$ for simple notation. Then we proceed as follows:

$$
\begin{gathered}
{[]_{1}=\left\{(1-\varepsilon) t_{1}^{2}+\sum_{i=2}^{m}(3-\varepsilon) t_{i}^{2}+\sum_{i=m+1}^{n-1} \frac{2 \lambda_{i}}{\lambda-\lambda_{i}} t_{i}^{2}\right\}+\frac{2 \lambda_{n}}{\lambda-\lambda_{n}} t_{n}^{2}} \\
=\left\{(1-\varepsilon) t_{1}^{2}+\sum_{i=2}^{m}(3-\varepsilon) t_{i}^{2}+\sum_{i=m+1}^{n-1} \frac{2 \lambda_{i}}{\lambda-\lambda_{i}} t_{i}^{2}\right\}+\frac{2 \lambda_{n}}{\lambda-\lambda_{n}}\left(\sum_{i=1}^{n-1} t_{i}\right)^{2} \\
\geq\left\{(1-\varepsilon) t_{1}^{2}+\sum_{i=2}^{m}(3-\varepsilon) t_{i}^{2}+\sum_{i=m+1}^{n-1} \frac{2 \lambda_{i}}{\lambda-\lambda_{i}} t_{i}^{2}\right\} . \\
{\left[1+\frac{2 \lambda_{n}}{\lambda-\lambda_{n}}\left(\frac{1}{1-\varepsilon}+\sum_{i=2}^{m} \frac{1}{3-\varepsilon}+\sum_{i=m+1}^{n-1} \frac{\lambda-\lambda_{i}}{2 \lambda_{i}}\right)\right],}
\end{gathered}
$$


where we used (2.12) and a Cauchy-Schartz inequality to reach the above inequality. We now show the second factor [] in the last term is also nonnegative:

$$
\begin{aligned}
& {\left[1+\frac{2 \lambda_{n}}{\lambda-\lambda_{n}}\left(\frac{1}{1-\varepsilon}+\sum_{i=2}^{m} \frac{1}{3-\varepsilon}+\sum_{i=m+1}^{n-1} \frac{\lambda-\lambda_{i}}{2 \lambda_{i}}\right)\right]} \\
& =\frac{2 \lambda_{n}}{\lambda-\lambda_{n}}\left(\frac{\lambda-\lambda_{n}}{2 \lambda_{n}}+\frac{1}{1-\varepsilon}+\frac{m-1}{3-\varepsilon}+\frac{\lambda-\lambda_{m+1}}{2 \lambda_{m+1}}+\cdots+\frac{\lambda-\lambda_{n-1}}{2 \lambda_{n-1}}\right) \\
& =\frac{2 \lambda_{n}}{\lambda-\lambda_{n}}\left[\frac{1}{1-\varepsilon}+\frac{m-1}{3-\varepsilon}+\frac{\lambda}{2}\left(\frac{1}{\lambda_{1}}+\cdots+\frac{1}{\lambda_{1}}\right)-\frac{n}{2}\right] \\
& =\frac{2 \lambda_{n}}{\lambda-\lambda_{n}}\left[\frac{1}{1-\varepsilon}+\frac{m-1}{3-\varepsilon}+\frac{\lambda}{2} \frac{\sigma_{n-1}}{\sigma n}-\frac{n}{2}\right] \\
& \geq \frac{2 \lambda_{n}}{\lambda-\lambda_{n}}\left(\frac{1}{1-\varepsilon}+\frac{m-1}{3-\varepsilon}-\frac{n}{2}\right) \\
& \geq 0,
\end{aligned}
$$

where we used $\lambda_{1}=\cdots=\lambda_{m}$, (2.4), and $\frac{1}{1-\varepsilon}+\frac{m-1}{3-\varepsilon}-\frac{n}{2} \leq 0$ under the assumption

$$
\varepsilon \leq 2-\frac{m}{n}-\sqrt{\left(1-\frac{m}{n}\right)^{2}+\frac{4}{n}} .
$$

Therefor []$_{1} \geq 0$.

Step 2.2. For each $k$ between $m$ and $n$, we have $\lambda_{k}>0$, the []$_{k}$ term in (2.11) satisfies

$$
\begin{aligned}
{[]_{k} } & =\sum_{i \leq m}\left(\frac{\lambda+\lambda_{k}}{\lambda-\lambda_{k}}-\varepsilon\right) t_{i}^{2} \\
& \geq \sum_{i \leq m}(1-\varepsilon) t_{i}^{2} \geq 0
\end{aligned}
$$

as long as $\varepsilon \leq 1$.

For $k=n$, the []$_{n}$ term in (2.11) becomes

$$
\begin{aligned}
{[]_{n} } & =\sum_{i \leq m}\left(\frac{\lambda+\lambda_{n}}{\lambda-\lambda_{n}}-\varepsilon\right) t_{i}^{2} \\
& \geq \sum_{i \leq m}\left(\frac{n-2}{n}-\varepsilon\right) t_{i}^{2} \geq 0
\end{aligned}
$$

where we used (2.3) and we also assumed $\varepsilon \leq \frac{n-2}{n}$.

Note that for $n-1 \geq m \geq 1$

$$
1-\frac{4}{\sqrt{4 n+1}+1} \leq 2-\frac{m}{n}-\sqrt{\left(1-\frac{m}{n}\right)^{2}+\frac{4}{n}} \leq \frac{n-2}{n},
$$


therefore we have proved (2.9) with $n-1 \geq m \geq 1$. When $m=n$, we have $\lambda_{1}=\cdots=\lambda_{n}>0$. Then from (2.10) we see in a much easier way that (2.9) holds.

The proof of Lemma 2.3 is complete.

Proposition 2.1. Let $u$ be a smooth solution to the special Lagrangian equation (1.1) with $n \geq 2$ and $\Theta \geq(n-2) \pi / 2$ on $B_{R}(0) \subset \mathbb{R}^{n}$. Set

$$
b=\ln \sqrt{1+\lambda_{\max }^{2}},
$$

where $\lambda_{\max }$ is the largest eigenvalue of Hessian $D^{2} u$, namely, $\lambda_{\max }=\lambda_{1} \geq$ $\cdots \geq \lambda_{n}$. Then $b$ satisfies the integral Jacobi inequality

$$
\int_{B_{R}}-\left\langle\nabla_{g} \varphi, \nabla_{g} b\right\rangle_{g} d v_{g} \geq \varepsilon(n) \int_{B_{R}} \varphi\left|\nabla_{g} b\right|^{2} d v_{g}
$$

for all non-negative $\varphi \in C_{0}^{\infty}\left(B_{R}\right)$, where $\varepsilon(n)=1-4 /(\sqrt{4 n+1}+1)$.

Proof. If $b(x)=b_{1}(x)$ is smooth everywhere, then the pointwise Jacobi inequality (2.9) in Lemma 2.3 with $m=1$ already implies the integral Jacobi inequality (2.13). In general, we know that $\lambda_{\max }$ is only a Lipschitz function of the entries of the Hessian $D^{2} u$. By the assumption, $D^{2} u(x)$ is smooth in $x$, thus $b=b_{1}=\ln \sqrt{1+\lambda_{\max }^{2}}$ is Lipschitz in terms of $x$.

Set $\varepsilon=\varepsilon(n)$. We first show that

$$
\triangle_{g} b \geq \varepsilon\left|\nabla_{g} b\right|^{2} \quad \text { in the viscosity sense. }
$$

Given any quadratic polynomial $Q$ touching $b$ from above at $p$. If $p$ is a smooth point of $b$, by (2.9) with $m=1$, we get

$$
\triangle_{g} Q \geq \varepsilon\left|\nabla_{g} Q\right|^{2} \quad \text { at } p .
$$

Otherwise, eigenvalue $\lambda_{1}$ is not distinct at $p$. Suppose $\lambda_{1}=\cdots=\lambda_{k}>\lambda_{k+1}$ at $p$. Then $Q$ also touches the smooth $b_{k}=\left(\ln \sqrt{1+\lambda_{1}^{2}}+\cdots+\ln \sqrt{1+\lambda_{k}^{2}}\right) / k$ from above at $p$, because

$$
b(x) \geq b_{k}(x) \text { and } b(p)=b_{k}(p) .
$$

By pointwise Jacobi inequality (2.9) with $m=k$, we still have

$$
\triangle_{g} Q \geq \varepsilon\left|\nabla_{g} Q\right|^{2} \quad \text { at } p \text {. }
$$

Next we switch to $a=e^{-\varepsilon b}$ and $a_{k}=e^{-\varepsilon b_{k}}$, the above argument leads to

$$
\triangle_{g} a \leq 0 \text { in the viscosity sense. }
$$

Relying on the definition of viscosity supersolutions, we see $a$ is $\triangle_{g}$-superharmonic in the potential sense, namely, $a \geq h$ in any regular domain $\Omega$ for $\triangle_{g^{-}}$ harmonic function $h$ with the boundary value $a$ on $\partial \Omega$ :

$$
\left\{\begin{array}{ll}
\triangle_{g} h=0 & \text { in } \Omega \\
h=a & \text { on } \partial \Omega
\end{array} .\right.
$$

By [HH, Theorem 1] (see also [Wn, p. 246]), we obtain

$$
\triangle_{g} a \leq 0 \text { in the distribution sense. }
$$


Note $a$ is Lipschitz because $b$ is. We move to the integral Jacobi inequality as follows. Take the test function $\varphi e^{\varepsilon b}$ for and nonnegative $\varphi \in C_{0}^{\infty}$, we get

$$
\begin{aligned}
0 & \geq \int_{B_{R}} \varphi e^{\varepsilon b} \triangle_{g} a d v_{g}=\int_{B_{R}}-\left\langle\nabla_{g}\left(\varphi e^{\varepsilon b}\right), \nabla_{g} a\right\rangle_{g} d v_{g} \\
& =\int_{B_{R}}\left\langle e^{\varepsilon b}\left(\nabla_{g} \varphi+\varepsilon \varphi \nabla_{g} b\right), \varepsilon e^{-\varepsilon b} \nabla_{g} b\right\rangle_{g} d v_{g} \\
& =\int_{B_{R}}\left(\varepsilon\left\langle\nabla_{g} \varphi, \nabla_{g} b\right\rangle_{g}+\varepsilon^{2} \varphi\left|\nabla_{g} b\right|_{g}^{2}\right) d v_{g} .
\end{aligned}
$$

Thus we arrive at the integral Jacobi inequality (2.13).

\section{Proof Of Theorem 1.1}

We assume that $R=2 n+1$ and $u$ is a solution on $B_{2 n+1} \subset \mathbb{R}^{n}$ for simplicity of notation. By scaling $v(x)=u\left(\frac{R}{2 n+1} x\right) /\left(\frac{R}{2 n+1}\right)^{2}$, we still get the estimate in Theorem 1.1. We consider the case $\Theta \geq(n-2) \pi / 2$. The negative phase case $\Theta \leq-(n-2) \pi / 2$ follows by symmetry.

Step 1. By the integral Jacobi inequality (2.13) in Proposition 2.1, $b$ is subharmonic in the integral sense. Then $b^{\frac{n}{n-2}}$ is also subharmonic in the integral sense on the minimal surface $\mathfrak{M}=(x, D u)$ :

$$
\begin{aligned}
& \int-\left\langle\nabla_{g} \varphi, \nabla_{g} b^{\frac{n}{n-2}}\right\rangle_{g} d v_{g} \\
& =\int-\left\langle\nabla_{g}\left(\frac{n}{n-2} b^{\frac{2}{n-2}} \varphi\right)-\frac{2 n}{(n-2)^{2}} b^{\frac{4-n}{n-2}} \varphi \nabla_{g} b, \nabla_{g} b\right\rangle_{g} d v_{g} \\
& \geq \int\left(\frac{n}{n-2} \varepsilon(n) \varphi b^{2}\left|\nabla_{g} b\right|^{2}+\frac{2 n}{(n-2)^{2}} b^{\frac{4-n}{n-2}} \varphi\left|\nabla_{g} b\right|^{2}\right) d v_{g} \geq 0
\end{aligned}
$$

for all non-negative $\varphi \in C_{0}^{\infty}$, where we approximate $b$ by smooth functions if necessary.

Applying Michael-Simon's mean value inequality [MS, Theorem 3.4] to the Lipschitz subharmonic function $b^{\frac{n}{n-2}}$, we obtain

$$
b(0) \leq C(n)\left(\int_{\mathfrak{B}_{1} \cap \mathfrak{M}} b^{\frac{n}{n-2}} d v_{g}\right)^{\frac{n-2}{n}} \leq C(n)\left(\int_{B_{1}} b^{\frac{n}{n-2}} d v_{g}\right)^{\frac{n-2}{n}},
$$

where $\mathfrak{B}_{r}$ is the ball with radius $r$ and center at $(0, D u(0))$ in $\mathbb{R}^{n} \times \mathbb{R}^{n}$, and $B_{r}$ is the ball with radius $r$ and center at 0 in $\mathbb{R}^{n}$. Choose a cut-off function $\varphi \in C_{0}^{\infty}\left(B_{2}\right)$ such that $\varphi \geq 0, \varphi=1$ on $B_{1}$, and $|D \varphi| \leq 1.1$; we then have

$$
\left(\int_{B_{1}} b^{\frac{n}{n-2}} d v_{g}\right)^{\frac{n-2}{n}} \leq\left(\int_{B_{2}} \varphi^{\frac{2 n}{n-2}} b^{\frac{n}{n-2}} d v_{g}\right)^{\frac{n-2}{n}}=\left(\int_{B_{2}}\left(\varphi b^{1 / 2}\right)^{\frac{2 n}{n-2}} d v_{g}\right)^{\frac{n-2}{n}} .
$$


Applying the Sobolev inequality on the minimal surface $\mathfrak{M}[\mathrm{MS}$, Theorem 2.1] or [A, Theorem 7.3] to $\varphi b^{1 / 2}$, which we may assume to be $C^{1}$ by approximation, we obtain

$$
\left(\int_{B_{2}}\left(\varphi b^{1 / 2}\right)^{\frac{2 n}{n-2}} d v_{g}\right)^{\frac{n-2}{n}} \leq C(n) \int_{B_{2}}\left|\nabla_{g}\left(\varphi b^{1 / 2}\right)\right|^{2} d v_{g}
$$

Decomposing the integrand as follows

$$
\begin{aligned}
\left|\nabla_{g}\left(\varphi b^{1 / 2}\right)\right|^{2} & =\left|\frac{1}{2 b^{1 / 2}} \varphi \nabla_{g} b+b^{1 / 2} \nabla_{g} \varphi\right|^{2} \leq \frac{1}{2 b} \varphi^{2}\left|\nabla_{g} b\right|^{2}+2 b\left|\nabla_{g} \varphi\right|^{2} \\
& \leq \frac{1}{\ln (4 / 3)} \varphi^{2}\left|\nabla_{g} b\right|^{2}+2 b\left|\nabla_{g} \varphi\right|^{2},
\end{aligned}
$$

where we used

$$
b \geq \ln \sqrt{1+\tan ^{2}\left(\frac{\pi}{2}-\frac{\pi}{n}\right)} \geq \ln \sqrt{4 / 3}
$$

we get

$$
\begin{aligned}
b(0) & \leq C(n) \int_{B_{2}}\left|\nabla_{g}\left(\varphi b^{1 / 2}\right)\right|^{2} d v_{g} \\
& \leq C(n)\left(\int_{B_{2}} \varphi^{2}\left|\nabla_{g} b\right|^{2} d v_{g}+\int_{B_{2}} b\left|\nabla_{g} \varphi\right|^{2} d v_{g}\right) .
\end{aligned}
$$

Step 2. By (2.13) in Proposition 2.1, $b$ satisfies the Jacobi inequality in the integral sense:

$$
\frac{1}{\varepsilon(n)} \triangle_{g} b \geq\left|\nabla_{g} b\right|^{2}
$$

Multiplying both sides by the above non-negative cut-off function $\varphi \in$ $C_{0}^{\infty}\left(B_{2}\right)$, then integrating, we obtain

$$
\begin{aligned}
\int_{B_{2}} \varphi^{2}\left|\nabla_{g} b\right|^{2} d v_{g} & \leq \frac{1}{\varepsilon(n)} \int_{B_{2}} \varphi^{2} \triangle_{g} b d v_{g} \\
& =\frac{-1}{\varepsilon(n)} \int_{B_{2}}\left\langle 2 \varphi \nabla_{g} \varphi, \nabla_{g} b\right\rangle d v_{g} \\
& \leq \frac{1}{2} \int_{B_{2}} \varphi^{2}\left|\nabla_{g} b\right|^{2} d v_{g}+\frac{2}{\varepsilon(n)^{2}} \int_{B_{2}}\left|\nabla_{g} \varphi\right|^{2} d v_{g}
\end{aligned}
$$

It follows that

$$
\int_{B_{2}} \varphi^{2}\left|\nabla_{g} b\right|^{2} d v_{g} \leq \frac{4}{\varepsilon(n)^{2}} \int_{B_{2}}\left|\nabla_{g} \varphi\right|^{2} d v_{g}
$$


So far we have reached

$$
\begin{aligned}
b(0) & \leq C(n)\left(\int_{B_{2}}\left|\nabla_{g} \varphi\right|^{2} d v_{g}+\int_{B_{2}} b\left|\nabla_{g} \varphi\right|^{2} d v_{g}\right) \\
& \leq C(n) \int_{B_{2}} b\left|\nabla_{g} \varphi\right|^{2} d v_{g} \\
& \leq C(n) \int_{B_{2}} b \sum_{i=1}^{n} \frac{1}{1+\lambda_{i}^{2}} \sqrt{\operatorname{det} g} d x,
\end{aligned}
$$

where in the second inequality, we again used $b \geq \ln \sqrt{4 / 3}$.

Step 3. Differentiating the complex identity

$$
\begin{aligned}
\ln V+\sqrt{-1} \sum_{i=1}^{n} \arctan \lambda_{i} & =\ln \prod_{i=1}^{n}\left(1+\sqrt{-1} \lambda_{i}\right) \\
& =\ln \left[\sum_{0 \leq 2 k \leq n}(-1)^{k} \sigma_{2 k}+\sqrt{-1} \sum_{1 \leq 2 k+1 \leq n}(-1)^{k} \sigma_{2 k+1}\right] .
\end{aligned}
$$

we obtain the (conformality) identity

$$
\left(\frac{1}{1+\lambda_{1}^{2}}, \cdots, \frac{1}{1+\lambda_{n}^{2}}\right) V=\left(\frac{\partial \Sigma}{\partial \lambda_{1}}, \cdots, \frac{\partial \Sigma}{\partial \lambda_{n}}\right)
$$

with $V=\sqrt{\operatorname{det} g}$ and

$$
\begin{aligned}
\Sigma & =\cos \Theta \sum_{1 \leq 2 k+1 \leq n}(-1)^{k} \sigma_{2 k+1}-\sin \Theta \sum_{0 \leq 2 k \leq n}(-1)^{k} \sigma_{2 k} \\
& =\sigma_{n-1}-\sigma_{n-3}+\cdots, \text { in particular when }|\Theta|=(n-2) \frac{\pi}{2} .
\end{aligned}
$$

Taking trace, we then get

$$
\begin{gathered}
\sum_{i=1}^{n} \frac{1}{1+\lambda_{i}^{2}} V=\sum_{i=1}^{n} \frac{\partial \Sigma}{\partial \lambda_{i}} \\
=\cos \Theta \sum_{1 \leq 2 k+1 \leq n}(-1)^{k}(n-2 k) \sigma_{2 k}-\sin \Theta \sum_{0 \leq 2 k \leq n}(-1)^{k}(n-2 k+1) \sigma_{2 k-1} \\
=c_{0}+c_{1} \sigma_{1}+\cdots+c_{n-1} \sigma_{n-1},
\end{gathered}
$$

where the coefficient $c_{i}$ depends only on $i, n$, and $\Theta$. At the critical phase $|\Theta|=(n-2) \pi / 2$, the leading term in (3.2) is $\sigma_{n-2}$

$$
\sum_{i=1}^{n} \frac{1}{1+\lambda_{i}^{2}} V=2 \sigma_{n-2}-4 \sigma_{n-4}+\cdots .
$$

In turn, (3.1) becomes

$$
b(0) \leq C(n) \int_{B_{2}} b\left(c_{0}+c_{1} \sigma_{1}+\cdots+c_{n-1} \sigma_{n-1}\right) d x .
$$


Step 4. Next we estimate the integrals $\int b \sigma_{k} d x$ for $1 \leq k \leq n-1$ inductively, using the divergence structure of $\sigma_{k}\left(D^{2} u\right)$ :

$$
\begin{aligned}
k \sigma_{k}\left(D^{2} u\right) & =\sum_{i, j=1}^{n} \frac{\partial \sigma_{k}}{\partial u_{i j}} \frac{\partial^{2} u}{\partial x_{i} \partial x_{j}}=\sum_{i, j=1}^{n} \frac{\partial}{\partial x_{i}}\left(\frac{\partial \sigma_{k}}{\partial u_{i j}} \frac{\partial u}{\partial x_{j}}\right) \\
& =\operatorname{div}\left(L_{\sigma_{k}} D u\right)
\end{aligned}
$$

where $L_{\sigma_{k}}$ denotes the matrix $\left(\frac{\partial \sigma_{k}}{\partial u_{i j}}\right)$. Let $\psi$ be a smooth cut-off function on $B_{\rho+1}$ such that $\psi=1$ on $B_{\rho}, 0 \leq \psi \leq 1$, and $|D \psi| \leq 1$.1. Noticing that $\sigma_{k}>0$ by (2.4) in Lemma 2.1 and $b>0$, we have

$$
\begin{gathered}
\int_{B_{\rho}} b \sigma_{k} d x \leq \int_{B_{\rho+1}} \psi b \sigma_{k} d x=\int_{B_{\rho+1}} \psi b \frac{1}{k} \operatorname{div}\left(L_{\sigma_{k}} D u\right) d x \\
=\frac{1}{k} \int_{B_{\rho+1}}-\left\langle b D \psi+\psi D b, L_{\sigma_{k}} D u\right\rangle d x \\
\leq C(n)\|D u\|_{L^{\infty}\left(B_{\rho+1}\right)}\left[\begin{array}{l}
\int_{B_{\rho+1}} b \sigma_{k-1} d x+ \\
\int_{B_{\rho+1}}\left[\left|\nabla_{g} b\right|^{2}+\operatorname{tr}\left(g^{i j}\right)\right] \sqrt{\operatorname{det} g} d x
\end{array}\right] .
\end{gathered}
$$

The last inequality was derived as follows. As all the above integrands are invariant under orthogonal transformations, at any point $p \in B_{\rho+1}$, we assume $D^{2} u(p)$ is diagonalized. Then $L_{\sigma_{k}}$ is also diagonal with positive entries $\partial_{\lambda_{i}} \sigma_{k}$. The positivity can be seen by applying Lemma 2.1 to all $\lambda_{1}, \cdots, \lambda_{n}$ but $\lambda_{i}$, whose corresponding phase is no less than $(n-3) \pi / 2$. Thus $0<\partial_{\lambda_{i}} \sigma_{k}<(n-k+1) \sigma_{k-1}$. Now we have

$$
\begin{aligned}
& \left|\left\langle b D \psi+\psi D b, L_{\sigma_{k}} D u\right\rangle\right| \stackrel{p}{\leq} \sum_{i=1}^{n}\left(b\left|D_{i} \psi\right|+\psi\left|D_{i} b\right|\right) \partial_{\lambda_{i}} \sigma_{k}\left|D_{i} u\right| \\
& \stackrel{p}{\leq} C(n)|D u(p)|\left(b \sigma_{k-1}+\sum_{i=1}^{n}\left|D_{i} b\right| \partial_{\lambda_{i}} \sigma_{k}\right) .
\end{aligned}
$$

Recall $k \leq n-1$, then $\partial_{\lambda_{i}} \sigma_{k}$ only consists of multiples of at most $(n-2)$ eigenvalues without $\lambda_{i}$. "Twist" multiplying the two $g^{\curvearrowright \odot}$ terms involving the missed $\lambda_{i}$ and the other eigenvalue, we obtain

$$
\begin{aligned}
& \left|D_{i} b\right| \partial_{\lambda_{i}} \sigma_{k} \stackrel{p}{\leq}\left|D_{i} b\right| \partial_{\lambda_{i}} \sigma_{k}\left(\left|\lambda_{1}\right|, \cdots,\left|\lambda_{n}\right|\right) \\
& \quad \stackrel{p}{\leq} C(n) \sum_{\alpha \neq i}\left(\frac{\left|D_{i} b\right|^{2}}{1+\lambda_{i}^{2}}+\frac{1}{1+\lambda_{\alpha}^{2}}\right) \sqrt{\left(1+\lambda_{1}^{2}\right) \cdots\left(1+\lambda_{n}^{2}\right)} .
\end{aligned}
$$

Summing up, we get

$$
\begin{aligned}
& \sum_{i=1}^{n}\left|D_{i} b\right| \partial_{\lambda_{i}} \sigma_{k} \stackrel{p}{\leq} C(n) \sum_{i=1}^{n}\left(g^{i i}\left|D_{i} b\right|^{2}+g^{i i}\right) \sqrt{\operatorname{det} g} \\
& \stackrel{p}{=} C(n)\left[\left|\nabla_{g} b\right|^{2}+\operatorname{tr}\left(g^{i j}\right)\right] \sqrt{\operatorname{det} g}
\end{aligned}
$$


The inequality (3.5) has been established. To simplify the last integral in (3.5), we repeat the integral Jacobi argument in Step 2 to get

$$
\int_{B_{\rho+1}}\left|\nabla_{g} b\right|^{2} \sqrt{\operatorname{det} g} d x \leq C(n) \int_{B_{\rho+2}} \operatorname{tr}\left(g^{i j}\right) \sqrt{\operatorname{det} g} d x .
$$

Hence (3.5) becomes the following inductive inequality

$$
\int_{B_{\rho}} b \sigma_{k} d x \leq C(n)\|D u\|_{L^{\infty}\left(B_{\rho+1}\right)}\left[\int_{B_{\rho+1}} b \sigma_{k-1} d x+\int_{B_{\rho+2}} \operatorname{tr}\left(g^{i j}\right) \sqrt{\operatorname{det} g} d x\right] .
$$

Step 4.1. We iterate (3.6) to derive

$$
\begin{gathered}
\int_{B_{2}} b \sigma_{k} d x \\
\leq C(n)\left\{\begin{array}{l}
\|D u\|_{L^{\infty}\left(B_{2+k}\right)}^{k} \int_{B_{2+k}} b d x+ \\
{\left[\|D u\|_{L^{\infty}\left(B_{2+k}\right)}^{k}+\cdots+\|D u\|_{L^{\infty}\left(B_{2+k}\right)}\right] \int_{B_{2+k+1}} \operatorname{tr}\left(g^{i j}\right) \sqrt{\operatorname{det} g} d x}
\end{array}\right\} \\
\leq C(n)\left\{\begin{array}{l}
\|D u\|_{L^{\infty}\left(B_{2+k}\right)}^{k+1}+ \\
{\left[\|D u\|_{L^{\infty}\left(B_{2+k}\right)}^{k}+\|D u\|_{L^{\infty}\left(B_{2+k}\right)}\right] \int_{B_{2+k+1}} \operatorname{tr}\left(g^{i j}\right) \sqrt{\operatorname{det} g} d x}
\end{array}\right\},
\end{gathered}
$$

where for the last inequality, we used Young's inequality and

$$
\int_{B_{2+k}} b d x \leq C(n)\|D u\|_{L^{\infty}\left(B_{2+k}\right)},
$$

which follows from

$$
b=\ln \sqrt{1+\lambda_{\max }^{2}}<\lambda_{\max }<\lambda_{1}+\lambda_{2}+\cdots+\lambda_{n}=\triangle u
$$

by (2.2) in Lemma 2.1. Putting all the estimates for $b \sigma_{k} \mathrm{~s}$ in (3.4) together, we get

$b(0) \leq C(n)\left\{\begin{array}{l}\|D u\|_{L^{\infty}\left(B_{n+1}\right)}^{n}+\|D u\|_{L^{\infty}\left(B_{n+1}\right)}+ \\ {\left[\|D u\|_{L^{\infty}\left(B_{n+1}\right)}^{n-1}+\|D u\|_{L^{\infty}\left(B_{n+1}\right)}\right] \int_{B_{n+2}} \operatorname{tr}\left(g^{i j}\right) \sqrt{\operatorname{det} g} d x}\end{array}\right\}$.

Step 4.2. We bound the last integral in the above inequality. Relying on the trace conformality identity (3.2), we derive

$$
\begin{aligned}
\int_{B_{n+2}} \operatorname{tr}\left(g^{i j}\right) \sqrt{\operatorname{det} g} d x & =\int_{B_{n+2}}\left(c_{0}+c_{1} \sigma_{1}+\cdots+c_{n-1} \sigma_{n-1}\right) d x \\
& \leq C(n)\left[\|D u\|_{L^{\infty}\left(B_{2 n+1}\right)}^{n-1}+1\right]
\end{aligned}
$$

where for the last inequality, we repeated the iteration integral estimates for (3.6) in Step 4.1 with $b=1$ (now much simpler)

$$
\int_{B_{\rho}} \sigma_{k} d x \leq C(n)\|D u\|_{L^{\infty}\left(B_{\rho+1}\right)} \int_{B_{\rho+1}} \sigma_{k-1} d x
$$


Finally from the above estimates (3.8) and (3.7), we conclude that

$$
b(0) \leq C(n)\left[\|D u\|_{L^{\infty}\left(B_{2 n+1}\right)}^{2 n-2}+\|D u\|_{L^{\infty}\left(B_{2 n+1}\right)}\right]
$$

and after exponentiating

$$
\left|D^{2} u(0)\right| \leq C(n) \exp \left[C(n)\|D u\|_{L^{\infty}\left(B_{2 n+1}\right)}^{2 n-2}\right] .
$$

Note at the critical phase $\Theta=(n-2) \pi / 2$, because of (3.3) , the leading term in (3.4) and (3.8) is $\sigma_{n-2}$. The iteration integral estimates in Step 4.1 and 4.2 start from $\sigma_{n-2}$. Thus we really obtain

$$
\left|D^{2} u(0)\right| \leq C(n) \exp \left[C(n)\|D u\|_{L^{\infty}\left(B_{2 n}\right)}^{2 n-4}\right] .
$$

The proof of Theorem 1.1 is complete.

\section{REFERENCES}

[A] Allard, William K., On the first variation of a varifold. Ann. of Math. (2) 95 (1972), 417-491.

[BC] Bao, Jiguang and Chen, Jingyi, Optimal regularity for convex strong solutions of special Lagrangian equations in dimension 3. Indiana Univ. Math. J. 52 (2003), 1231-1249.

[BCGJ] Bao, Jiguang, Chen, Jingyi, Guan, Bo, and Ji, Min, Amer. J. Math. 125 (2003), 301-316.

[BDM] Bombieri, Enrico, De Giorgi, Ennio and Miranda, Mario, Una maggiorazione a priori relativa alle ipersuperfici minimali non parametriche. Arch. Rational Mech. Anal. 32 (1969), 255-267.

[CNS] Caffarelli, Luis, Nirenberg, Louis, and Spruck, Joel, The Dirichlet problem for nonlinear second-order elliptic equations. III. Functions of the eigenvalues of the Hessian. Acta Math. 155 (1985), 261-301.

[CWY] Chen, Jingyi, Warren, Micah, and Yuan, Yu, A priori estimate for convex solutions to special Lagrangian Equations and its application. Comm. Pure Appl. Math. 62 (2009), no. 4, 583-595.

[CW] Chou, Kai-Seng and Wang, Xu-Jia, A variational theory of the Hessian equation. Comm. Pure Appl. Math. 54 (2001), 1029-1064.

[F] Finn, Robert, New estimates for equations of minimal surface type, Arch. Rational Mech. Anal. 14 (1963), 337-375.

[HL] Harvey, Reese and Lawson, H. Blaine. Jr., Calibrated geometry. Acta Math. 148 (1982), 47-157.

[H] Heinz, Erhard, On elliptic Monge-Ampère equations and Weyl's embedding problem. J. Analyse Math. 7 (1959) 1-52.

[HH] Hervé, Rose-Marie and Hervé, Michel, Les fonctions surharmoniques dans l'axiomatique de M. Brelot associées àun opéateur elliptique dégénéré. (French) Ann. Inst. Fourier (Grenoble) 22 (1972), no. 2, 131-145.

[J] Jögens, Konrad, Üer die Löungen der Differentialgleichung $r t-s^{2}=1$. (German) Math. Ann. 127 (1954), 130-134.

[K] N. Korevaar, An easy proof of the interior gradient bound for solutions to the prescribed mean curvature equation. Nonlinear functional analysis and its applications, Part 2 (Berkeley, Calif., 1983), 81-89, Proc. Sympos. Pure Math., 45, Part 2, Amer. Math. Soc., Providence, RI, 1986.

[MS] Michael, James H. and Simon, Leon M., Sobolev and mean-value inequalities on generalized submanifolds of $\mathbb{R}^{n}$. Comm. Pure Appl. Math. 26 (1973), 361-379. 
[NV] Nadirashvili, Nicolai and Vlăduţ, Serge, Singular solution to Special Lagrangian Equations. Ann. Inst. H. Poincaré Anal. Non Linéaire 27 (2010), no. 5, 1179-1188.

[P1] Pogorelov, Aleksei Vasil'evich, Monge-Ampère Equations of Elliptic Type. Translated from the first Russian edition by Leo F. Boron with the assistance of Albert L. Rabenstein and Richard C. Bollinger P. Noordhoff, Ltd., Groningen, 1964

[P2] Pogorelov, Aleksei Vasil'evich, The Minkowski Multidimensional Problem. Translated from the Russian by Vladimir Oliker. Introduction by Louis Nirenberg. Scripta Series in Mathematics. V. H. Winston \& Sons, Washington, D.C.; Halsted Press [John Wiley \& Sons], New York-Toronto-London, 1978.

[T1] Trudinger, Neil S. A new proof of the interior gradient bound for the minimal surface equation in $n$ dimensions. Proc. Nat. Acad. Sci. U.S.A. 69 (1972), 821823.

[T2] Trudinger, Neil S. Regularity of solutions of fully nonlinear elliptic equations. Boll. U.M.I. (6) 3-A (1984), 421-430.

[U1] Urbas, John I. E., On the existence of nonclassical solutions for two classes of fully nonlinear elliptic equations. Indiana Univ. Math. J. 39 (1990), no. 2, 355-382.

[U2] Urbas, John, Some interior regularity results for solutions of Hessian equations. Calc. Var. Partial Differential Equations 11 (2000), 1-31.

[U3] Urbas, John, An interior second derivative bound for solutions of Hessian equations. Calc. Var. Partial Differential Equations 12 (2001), 417-431.

[WdY] Wang, Dake and Yuan, Yu, Singular solutions to special Lagrangian equations with subcritical phases and minimal surface systems. Amer. J. Math., to appear.

[WY1] Warren, Micah and Yuan, Yu, A Liouville type theorem for special Lagrangian Equations with constraints. Comm. Partial Differential Equations 33 (2008), 922932.

[WY2] Warren, Micah and Yuan, Yu, Hessian estimates for the sigma-2 equation in dimension three. Comm. Pure Appl. Math. 62 (2009), no. 3, 305-321.

[WY3] Warren, Micah and Yuan, Yu, Explicit gradient estimates for minimal Lagrangian surfaces of dimension two. Math. Z. 262 (2009), no. 4, 867-879.

[WY4] Warren, Micah and Yuan, Yu, Hessian and gradient estimates for three dimensional special Lagrangian Equations with large phase. Amer. J. Math. 132 (2010), no. $3,751-770$.

[Wn] Watson, Neil A., Generalizations of the spherical mean convexity theorem on subharmonic functions. Ann. Acad. Sci. Fenn. Ser. A I Math. 17 (1992), no. 2, 241-255.

[Y1] Yuan, Yu, A Bernstein problem for special Lagrangian equations. Invent. Math. 150 (2002), 117-125.

[Y2] Yuan, Yu, Global solutions to special Lagrangian equations. Proc. Amer. Math. Soc. 134 (2006), no. 5, 1355-1358.

Department of Mathematics, Box 354350, University of Washington, SeatTLE, WA 98195

E-mail address: dkpool@math.washington.edu, yuan@math.washington.edu 